\title{
Representative families for matroid intersections, with applications to location, packing, and covering problems ${ }^{\star}$
}

\author{
René van Bevern ${ }^{\mathrm{a}, *}$, Oxana Yu. Tsidulko ${ }^{\mathrm{a}, \mathrm{b}}$, Philipp Zschoche ${ }^{\mathrm{c}}$ \\ ${ }^{a}$ Department of Mechanics and Mathematics, Novosibirsk State University, Novosibirsk, Russian Federation \\ ${ }^{b}$ Sobolev Institute of Mathematics of the Siberian Branch of the Russian Academy of Sciences, Novosibirsk, Russian Federation \\ ${ }^{c}$ Technische Universität Berlin, Faculty IV, Algorithmics and Computational Complexity, Germany
}

\begin{abstract}
We show algorithms for computing representative families for matroid intersections and use them in fixed-parameter algorithms for set packing, set covering, and facility location problems with multiple matroid constraints. We complement our tractability results by hardness results.
\end{abstract}

Keywords: combinatorial optimization, matroid set packing, matroid parity, matroid median

\section{Introduction}

Matroids are an important tool in the development of fixed-parameter algorithms [27] and many of these algorithms are based on the fast construction of so-called representative families [14-16, 23, 25, 31]. Generalizing these, we present algorithms to compute representative families not for single matroids, but for intersections of multiple matroids.

Using this generalization, we derive fixed-parameter algorithms for packing, covering and facility location problems with multiple matroid constraints. Herein, our algorithms for packing and covering problems generalize and unify several fixed-parameter algorithms for covering problems known in the literature [6, 24, 25]. In the context of facility location problems, matroid constraints can model natural facility location scenarios like "open at most $k_{i}$ facilities of type $i$ " [10], even if types are not disjoint, moving facilities [30], but also problems in social network analysis [5, Section 3].

Organization of this work. In Section 2 we provide basic definitions from parameterized complexity and matroid theory. Section 3 presents our algorithms for constructing representative families for matroid intersections. Sections 4 and 5 present our fixed-parameter algorithm for set packing and facility location problems with multiple matroid constraints, respectively. Related work and context for each result is provided in the respective subsections.

\section{Preliminaries}

\subsection{Parameterized complexity}

The main idea of fixed-parameter algorithms is to accept the exponential running time seemingly inherent to solving NP-hard problems, yet to confine the combinatorial explosion to a parameter of the problem, which can be small in applications [11]. A problem is fixed-parameter tractable if it can be solved in $f(k) \cdot \operatorname{poly}(n)$ time on inputs of length $n$ and some function $f$ depending only on some parameter $k$. Note that this requirement is stronger than an algorithm that merely runs in polynomial time for fixed $k$, say, in $O\left(n^{k}\right)$ time, which is inapplicable even for small values of $k$, say $k=10$. The parameterized analog of NP and NP-hardness is the $W$-hierarchy FPT $\subseteq W[1] \subseteq W[2] \subseteq \ldots W[P] \subseteq \mathrm{XP}$ and $W[t]$-hardness, where FPT is the class of fixed-parameter tractable decision problems and all inclusions are conjectured to be strict. If some $W[t]$-hard problem is in FPT, then FPT $=W[t][11]$.

\footnotetext{
^ The results in this work were announced in an extended abstract at CIAC 2019 [5].

${ }^{*}$ Correspondence to: Department of Mechanics and Mathematics, Novosibirsk State University, ul. Pirogova 1, Novosibirsk, 630090, Russian Federation

Email addresses: rvb@nsu.ru (René van Bevern), tsidulko@math.nsc.ru (Oxana Yu. Tsidulko), zschoche@tu-berlin.de (Philipp Zschoche)
} 


\subsection{Sets and set functions}

By $\mathbb{N}$, we denote the natural numbers including zero. By $\mathbb{F}_{p}$, we denote the field on $p$ elements. Usually, we study set systems over a universe $U=\{1, \ldots, n\}$. By $A \uplus B$, we denote the union of sets $A$ and $B$ that we require to be disjoint. By convention, the intersection of no sets is the whole universe and the union of no sets is the empty set.

Definition 2.1 (partition). We call $Z_{1}, \ldots, Z_{\ell}$ a partition of a set $A$ if $Z_{1} \uplus \ldots \uplus Z_{\ell}=A$ and $Z_{i} \neq \emptyset$ for each $i \in\{1, \ldots, \ell\}$.

Definition 2.2 ( $\gamma$-family). We call $A \subseteq 2^{U}$ an $\gamma$-family if each set in $A$ has cardinality exactly $\gamma$.

Definition 2.3 (additive and submodular set functions). A set function $w: 2^{U} \rightarrow \mathbb{R}$ is additive if, for any subsets $A \cup$ $B \subseteq U$, one has

$$
w(A \cup B)=w(A)+w(B)-w(A \cap B) .
$$

If " $\leq$ " holds instead of equality, then $w$ is called submodular.

Definition 2.4 (coverage function). A set function

$$
w: 2^{U} \rightarrow \mathbb{N}, S \mapsto\left|\bigcup_{u \in S} u\right|,
$$

where $U=2^{V}$, is a coverage function.

Coverage functions are non-decreasing and submodular [28, Section 44.1a].

\subsection{Matroid fundamentals}

For proofs of the following propositions and for illustrative examples of the following definitions, we refer to the book by Oxley [26].

Definition 2.5 (matroid). A pair $(U, \mathcal{I})$, where $U$ is the ground set and $\mathcal{I} \subseteq 2^{U}$ is a family of independent sets, is a matroid if the following holds:

- $\emptyset \in I$,

- If $A^{\prime} \subseteq A$ and $A \in \mathcal{I}$, then $A^{\prime} \in \mathcal{I}$.

- If $A, B \in \mathcal{I}$ and $|A|<|B|$, then there is an $x \in B \backslash A$ such that $A \cup\{x\} \in \mathcal{I}$.

Definition 2.6 (basis, rank). An inclusion-wise maximal independent set $A \in \mathcal{I}$ of a matroid $M=(U, \mathcal{I})$ is a basis. The cardinality of the bases of $M$ is called the rank of $M$.

Definition 2.7 (free matroid). A free matroid is a matroid $\left(U, 2^{U}\right)$ in which every set is independent.

Proposition 2.8 (matroid union, direct sum). The union

$$
M_{1} \vee M_{2}=\left(U_{1} \cup U_{2},\left\{J_{1} \cup J_{2} \mid J_{1} \in I_{1}, J_{2} \in I_{2}\right\}\right)
$$

of two matroids $M_{1}=\left(U_{1}, I_{1}\right)$ and $M_{2}=\left(U_{2}, I_{2}\right)$ is a matroid. If $U_{1} \cap U_{2}=\emptyset$, we write $M_{1} \oplus M_{2}:=M_{1} \vee M_{2}$ and call their union direct sum.

Definition 2.9 (uniform, partition, and multicolored matroids). A uniform matroid of rank $r$ is a matroid $(U, \mathcal{I})$ such that $\mathcal{I}:=\{S \subseteq U|| S \mid \leq r\}$. The direct sum of uniform matroids is called partition matroid. We call the direct sum of uniform matroids of rank one a multicolored matroid.

Partition matroids are useful to model constraints of type "at most $k_{i}$ items of type $i$ ". 


\subsection{Matroid representations}

In our work, we will use two different ways of representing matroids. The most general representation of matroids is an independence oracle, which in constant time decides whether a given set is independent in a given matroid. One can imagine it as an algorithm that decides independence quickly. We will also use linear representations:

Definition 2.10 (linear matroids). An $(r \times n)$-representation of a matroid $M=(U, \mathcal{I})$ over a field $\mathbb{F}$ is a matrix $A \in \mathbb{F}^{r \times n}$ whose columns are labeled by the nelements of $U$ such that $S \in \mathcal{I}$ if and only if the columns of $A$ with labels in $S$ are linearly independent over $\mathbb{F}$. A matroid is linear or representable over a field $\mathbb{F}$ if it has a representation over $\mathbb{F}$.

One can transform a representation of a matroid with rank $r$ over a field $\mathbb{F}$ into a representation over $\mathbb{F}$ with $r$ rows [26, Section 2.2] and we will always assume to work with linear representations of this form. Not all matroids are representable over all fields [26, Theorem 6.5.4]. Some are not representable over any field [26, Example 1.5.14]. If $A_{i}$ is a $\left(a_{i} \times b_{i}\right)$-representation of a matroid $M_{i}$ over field $\mathbb{F}$ for $i \in\{1, \ldots, m\}$, then a $\left(\sum_{i=1}^{m} a_{i} \times \sum_{i=1}^{m} b_{i}\right)$-representation of $\bigoplus_{i=1}^{m} M_{i}$ over $\mathbb{F}$ is computable in time of $O\left(\sum_{i=1}^{m} a_{i} \cdot \sum_{i=1}^{m} b_{i}\right)$ operations over $\mathbb{F}$ [26, Exercise 6, p. 132]. Uniform matroids of rank $r$ on a universe of size $n$ are representable over all fields with at least $n$ elements [25, Section 3.5]. The uniform matroid of rank one is trivially representable over all fields. Thus, so are multicolored matroids.

Lemma 2.11. Given an $(r \times n)$-representation A for a matroid $M$ over a field $\mathbb{F}$, a representation of $M \vee\left(X, 2^{X}\right)$ over $\mathbb{F}$ is computable in time of $(n+|X|)(r+|X|)$ operations over $\mathbb{F}$.

Proof. Let $M=(U, \mathcal{I})$. If $U \cap X \neq \emptyset$, consider the restriction $M^{\prime}=(U \backslash X,\{J \subseteq U \backslash X \mid J \in \mathcal{I}\})$ of $M$ to $U \backslash X$, which is again a matroid [26, Section 1.3]. If $U \cap X=\emptyset$, then we get $M^{\prime}=M$. A linear representation $A^{\prime}$ for $M^{\prime}$ can be obtained from a linear representation of $A$ for $M$ by removing the columns labeled by elements in $X$. The free matroid $\left(X, 2^{X}\right)$ is representable by the identity matrix over any field. Thus, $M \vee\left(X, 2^{X}\right)=M^{\prime} \vee\left(X, 2^{X}\right)=M^{\prime} \oplus\left(X, 2^{X}\right)$ is a direct sum of two matroids with known linear representations. The linear representation of this direct sum can therefore be easily obtained in the claimed time [26, Exercise 6, p. 132].

\subsection{Matroids truncations}

Definition 2.12 (truncation). The $k$-truncation of a matroid $(U, \mathcal{I})$ is a matroid $\left(U, \mathcal{I}^{\prime}\right)$ with $\mathcal{I}^{\prime}=\{S \subseteq U \mid S \in$ $\mathcal{I} \wedge|S| \leq k\}$. Moreover, if $A$ is a linear representation of a matroid and $A^{\prime}$ is a linear representation of its truncation, we will also call $A^{\prime}$ a truncation of $A$.

Proposition 2.13 (Lokshtanov et al. [23, Theorem 3.15]). Let $A$ be an $(r \times n)$-matrix of rank $r$ over a finite field $\mathbb{F}_{p^{d}}$, where $p$ is a prime number which is polynomially upper-bounded by the length of the encoding of $A$ as a binary string. For any $k \in\{1, \ldots, r\}$, we can compute a $k$-truncation of $A$ over a finite field extension $\mathbb{K} \supseteq \mathbb{F}$ in time of a polynomial number of field operations over $\mathbb{F}$, where $\mathbb{K}=\mathbb{F}_{p^{r k d} \text {. }}$

Remark 2.14. The proof of Lokshtanov et al. [23, Theorem 3.15] shows that the field extension $\mathbb{K} \supseteq \mathbb{F}:=\mathbb{F}_{p^{d}}$ in Proposition 2.13 can be chosen as $\mathbb{K}=\mathbb{F}_{p^{s d}}$ for any integer $s \geq r k$. The time for computing the truncation consists of computing a truncation over the field of fractions $\mathbb{F}(X)$ using $O(n k r)$ operations over $\mathbb{F}$ via Theorem 3.14 of Lokshtanov et al. [23] and then computing an irreducible polynomial of degree $s$ over $\mathbb{F}$ in $s^{4} d^{2} \sqrt{p} \cdot \operatorname{polylog}(s, p, d)$ operations over $\mathbb{F}[29]$.

Proposition 2.13 applied according to Remark 2.14 immediately yields:

Corollary 2.15. For $i \in\{1, \ldots, m\}$, let $A_{i}$ be $\left(r_{i} \times n\right)$-matrices over $\mathbb{F}_{p^{d}}$. Given a natural number $k \leq \min \left\{r_{i} \mid 1 \leq i \leq m\right\}$, $k$-truncations of the $A_{i}$ over the same finite field extension $\mathbb{F}_{p^{r k d}} \supseteq \mathbb{F}$ are computable in $O(m n k r)+r^{4} k^{4} d^{2} \sqrt{p}$. polylog $(r, k, p, d)$ operations over $\mathbb{F}$, where $r=\max \left\{r_{i} \mid 1 \leq i \leq m\right\}$.

Herein, the additive running time is due to the fact that we only have to construct the irreducible polynomial of degree $r k$ once in order to represent the truncated matroids over the same field extension $\mathbb{F}_{p^{r k d}}$. 


\section{Representative families for matroid intersections}

Intuitively, a representative of some set family $\mathcal{S}$ for a matroid $M$ is a subfamily $\widehat{\mathcal{S}} \subseteq \mathcal{S}$ such that, if $\mathcal{S}$ contains a set $X$ that can be extended to a basis of $M$ by adding adding a set $Y$, then $\widehat{\mathcal{S}}$ contains a set $\widehat{X}$ that can also be extended to a basis of $M$ by adding $Y$. Herein, the representative $\widehat{\mathcal{S}}$ may be significantly smaller than the original family $\mathcal{S}$, so that algorithms can gain a speed-up by working on $\widehat{\mathcal{S}}$ instead of $\mathcal{S}$.

Marx [25] first used representative families in randomized fixed-parameter algorithms for the NP-hard MATROID INTERSECTION problem, where the task is to decide whether a set is independent in several given matroids. Representative families have subsequently been generalized to weighted sets by Fomin et al. [14] and their construction has been derandomized by Lokshtanov et al. [23]. We generalize this concept to representative families for matroid intersections:

Definition 3.1 (max intersection $q$-representative family). Given matroids $\left\{\left(U, \mathcal{I}_{i}\right)\right\}_{i=1}^{m}$, a family $\mathcal{S} \subseteq 2^{U}$, and a function $w: \mathcal{S} \rightarrow \mathbb{R}$, we say that a subfamily $\widehat{\mathcal{S}} \subseteq \mathcal{S}$ is max intersection $q$-representative for $\mathcal{S}$ with respect to $w$ if, for each set $Y \subseteq U$ of size at most $q$, it holds that: if there is a set $X \in \mathcal{S}$ with $X \uplus Y \in \bigcap_{i=1}^{m} \mathcal{I}_{i}$, then there is a set $\widehat{X} \in \widehat{\mathcal{S}}$ with $\widehat{X} \uplus Y \in \bigcap_{i=1}^{m} I_{i}$ and $w(\widehat{X}) \geq w(X)$. If $m=1$, then we call $\widehat{\mathcal{S}} a$ max $q$-representative family of $\mathcal{S}$.

In this section, we will show how to compute max intersection representative families for matroids $\left\{\left(U, \mathcal{I}_{i}\right)\right\}_{i=1}^{m}$. More generally, for some $\mathcal{H} \subseteq 2^{U}$, we will compute max intersection representatives for subsets of the family

$$
\mathcal{B}(\mathcal{H}):=\left\{\biguplus_{j=1}^{i} H_{j} \mid i \in \mathbb{N}, H_{1}, \ldots, H_{i} \in \mathcal{H}\right\}
$$

in a time that will grow merely linearly in $|\mathcal{H}|$, whereas the size of $\mathcal{B}(\mathcal{H})$ is generally exponential in $|\mathcal{H}|$. For this to work, we require the weights of the sets in $\mathcal{B}(\mathcal{H})$ to be computable from weights of sets in $\mathcal{H}$ using inductive union maximizing functions, which we introduce in Section 3.1. In Section 3.2, we show how to compute representatives with respect to inductive union maximizing set functions in a single matroid. In Section 3.3, we generalize this result to max intersection representative families (for multiple matroids).

\subsection{Inductive union maximizing functions}

Definition 3.2 (inductive union maximizing function). Let $\mathcal{H} \subseteq 2^{U}$. A set function $\mathbf{w}: \mathcal{B}(\mathcal{H}) \rightarrow \mathbb{R}$ is called an inductive union maximizing function if there is a function $\mathbf{g}: \mathbb{R} \times \mathcal{H} \rightarrow \mathbb{R}$ that is non-decreasing in its first argument and such that, for each $X \neq \emptyset$,

$$
\mathbf{w}(X) \underset{\substack{H \in \mathcal{H}, S \in \mathcal{B}(\mathcal{H}) \\ S \cup H=X}}{\operatorname{man}(\mathbf{w}(S), H)}
$$

An inductive union maximizing function $\mathbf{w}$ is fully determined by the value $\mathbf{w}(\emptyset)$ and the function $\mathbf{g}$. We thus also say that $\mathbf{g}$ generates $\mathbf{w}$. Inductive union maximizing functions resemble primitive recursive functions on natural numbers, where $S \uplus H$ plays the role of the "successor" of $S$ in primitive recursion. We take the maximum over all partitions $S \uplus H=X$ since the partition of $X$ into a set in $\mathcal{H}$ and a set in $\mathcal{B}(\mathcal{H})$ is not unique. We now show some examples and counterexamples for inductive union maximizing functions.

Example 3.3. Let $\mathcal{H} \subseteq 2^{U}$ and $w: \mathcal{H} \rightarrow \mathbb{R}$. The function $\mathbf{w}$ determined by $\mathbf{w}(\emptyset)=0$ and $\mathbf{g}:(k, H) \mapsto k+w(H)$ is an inductive union maximizing function. Concretely, for $\emptyset \neq X \subseteq \mathcal{B}(\mathcal{H})$, one gets

$$
w_{\Sigma}(X):=\mathbf{w}(X)=\max _{\substack{X=H_{1} \uplus \uplus \cup H_{i} \\ H_{1}, \ldots, H_{i} \in \mathcal{H}}} \sum_{j=1}^{i} w\left(H_{j}\right)
$$

due to the associativity and commutativity of the maximum and sum.

Inductive union maximizing functions generalize additive set functions:

Example 3.4. Any additive set function $w: 2^{U} \rightarrow \mathbb{R}$ is inductive union maximizing since, for the inductive union maximizing function $w_{\Sigma}$ in Example 3.3, one has $w_{\Sigma}(X)=\sum_{j=1}^{i} w\left(H_{j}\right)=w(X)$ for any partition $X=H_{1} \uplus \cdots \uplus H_{j}$. 
However, submodular functions are generally not inductive union maximizing:

Example 3.5. Let $f: 2^{U} \rightarrow \mathbb{R}$ be a coverage function (cf. Definition 2.4 ) on $U=\left\{u_{1}, v_{1}, u_{2}, v_{2}\right\}$ with

$$
u_{1}=\{a\}, \quad v_{1}=\{c\}, \quad u_{2}=\{a, b\}, \quad \text { and } \quad v_{2}=\{c, d\} .
$$

Assume that $f$ is inductive union maximizing for $\mathcal{H}=\left\{\left\{u_{1}\right\},\left\{v_{1}\right\},\left\{u_{2}\right\},\left\{v_{2}\right\}\right\}$. The only partition of $\left\{u_{1}, v_{2}\right\}$ into sets in $\mathcal{H}$ is $\left\{u_{1}\right\} \uplus\left\{v_{2}\right\}$. Thus, $f\left(\left\{u_{1}, v_{2}\right\}\right)=\mathbf{g}\left(f\left(\left\{u_{1}\right\}\right),\left\{v_{2}\right\}\right)$ or $f\left(\left\{u_{1}, v_{2}\right\}\right)=\mathbf{g}\left(f\left(\left\{v_{2}\right\}\right),\left\{u_{1}\right\}\right)$, whichever is larger. In the first case, we get the contradiction

$$
3=f\left(\left\{u_{1}, v_{2}\right\}\right)=\mathbf{g}\left(f\left(\left\{u_{1}\right\}\right),\left\{v_{2}\right\}\right)=\mathbf{g}\left(1,\left\{v_{2}\right\}\right)=\mathbf{g}\left(f\left(\left\{v_{1}\right\}\right),\left\{v_{2}\right\}\right) \leq f\left(\left\{v_{1}, v_{2}\right\}\right)=2 .
$$

Otherwise, in the second case, we get the contradiction

$$
3=f\left(\left\{u_{1}, v_{2}\right\}\right)=\mathbf{g}\left(f\left(\left\{v_{2}\right\}\right),\left\{u_{1}\right\}\right)=\mathbf{g}\left(1,\left\{u_{1}\right\}\right)=\mathbf{g}\left(f\left(\left\{u_{2}\right\}\right),\left\{u_{1}\right\}\right) \leq f\left(\left\{u_{1}, u_{2}\right\}\right)=2 .
$$

We see that coverage functions are not inductive union maximizing since the function $\mathbf{g}$ generating $\mathbf{w}$ in Definition 3.2 is allowed to depend only on $\mathbf{w}(S)$ in the first argument, not on $S$ itself. We will indeed see that this requirement is crucial and presume that inductive union maximizing functions are the most general class of functions with respect to which we can prove the results in Sections 3.2 and 3.3.

\subsection{Computing representative families for unions of disjoint sets}

In this section, we show how to compute a representative of subfamilies of $\mathcal{B}(\mathcal{H})$ with respect to inductive union maximizing set functions in a single matroid. We generalize it to multiple matroids in Section 3.3.

Proposition 3.6. Let $M=(U, \mathcal{I})$ be a linear matroid of rank $r=(\alpha+\beta) \gamma \geq 1$ with $\alpha, \beta \in \mathbb{N}, \mathcal{H} \subseteq 2^{U}$ be a $\gamma$-family of size $t$, and $\mathbf{w}: \mathcal{B}(\mathcal{H}) \rightarrow \mathbb{R}$ be an inductive union maximizing function (cf. Definition 3.2) generated by $\mathbf{w}(\emptyset)$ and the function $\mathbf{g}: \mathbb{R} \times \mathcal{H} \rightarrow \mathbb{R}$ non-decreasing in the first argument.

Given $\alpha \in \mathbb{N}$, a representation A of $M$ over a field $\mathbb{F}$, the value $\mathbf{w}(\emptyset)$, and the function $\mathbf{g}$, one can compute a max $\beta \gamma$-representative $\widehat{\mathcal{S}}$ of size $\left(\begin{array}{c}r \\ \alpha \gamma\end{array}\right)$ for the family

$$
\mathcal{S}=\left\{S=H_{1} \uplus \cdots \uplus H_{\alpha} \mid S \in \mathcal{I} \text { and } H_{j} \in \mathcal{H} \text { for } j \in\{1, \ldots, \alpha\}\right\}
$$

with respect to $\mathbf{w}$ in time of $O\left(2^{\omega r} \cdot t\right)$ operations over $\mathbb{F}$ and calls to the function $\mathbf{g}$, where $\omega \geq 2$ is any constant such that two $(n \times n)$-matrices can be multiplied in $O\left(n^{\omega^{\prime}}\right)$ time for $\omega^{\prime}<\omega$.

Before proving Proposition 3.6, we provide some context. The main feature of Proposition 3.6 is that it allows us to compute max intersection representatives of the family $\mathcal{S}$, whose size may be exponential in the size of $\mathcal{H}$, in time growing merely linearly in the size of $\mathcal{H}$. The literature uses several implicit ad-hoc proofs of variants of Proposition 3.6 in algorithms for concrete problems $[14,15,25]$. These proofs usually use non-negative additive functions in place of w. Our Proposition 3.6 does not require additivity, yet, as shown by Example 3.4, works perfectly fine for all additive weight functions.

As shown by Example 3.5, submodular functions are not necessarily inductive union maximizing and, indeed, we now show that generalizing Proposition 3.6 even to coverage functions would yield FPT $=W[2]$. The proof also makes for an illustration of Definition 3.1 and Proposition 3.6:

Observation 3.7. If Proposition 3.6 holds for coverage functions $\mathbf{w}$, then FPT $=W[2]$.

Proof. Consider a coverage function $\mathbf{w}: 2^{U} \rightarrow \mathbb{N}$. The problem of finding a set $S \subseteq U$ with $|S| \leq r$ maximizing $\mathbf{w}(S)$ is known as Maximum Coverage and W[2]-hard parameterized by $r$ [11].

Now, assume that we could apply Proposition 3.6 with a uniform matroid $M=(U, \mathcal{I})$ of rank $r, \alpha=r, \beta=0, \gamma=1$, and the $\gamma$-family $\mathcal{H}=\{\{u\} \mid u \in U\}$ to compute a 0 -representative $\widehat{\mathcal{S}}$ of size $\left(\begin{array}{c}r \\ \alpha \gamma\end{array}\right)=1$ of the family

$$
\mathcal{S}=\left\{S=H_{1} \uplus \cdots \uplus H_{\alpha} \mid S \in \mathcal{I} \text { and } H_{j} \in \mathcal{H} \text { for } j \in\{1, \ldots, \alpha\}\right\}=\{S \subseteq U|| S \mid=r\}
$$

with respect to $\mathbf{w}$ in time of $2^{O(r)} \cdot|\mathcal{H}|=2^{O(r)} n$ operations over $\mathbb{F}$, where $M$ is representable over $\mathbb{F}=\mathbb{F}_{2^{d}}$ for $d=\lceil\log r\rceil$. Then $\widehat{\mathcal{S}}$ only contains the set $S$ with $|S|=r$ maximizing $\mathbf{w}(S)$. Since each operation over $\mathbb{F}_{2^{d}}$ can be carried out in $\operatorname{poly}(d) \in \operatorname{poly}(n)$ time, we thus solve the $W[2]$-hard Maximum Coverage problem in $2^{O(r)} \cdot \operatorname{poly}(n)$ time, which implies $\mathrm{FPT}=W[2]$. 


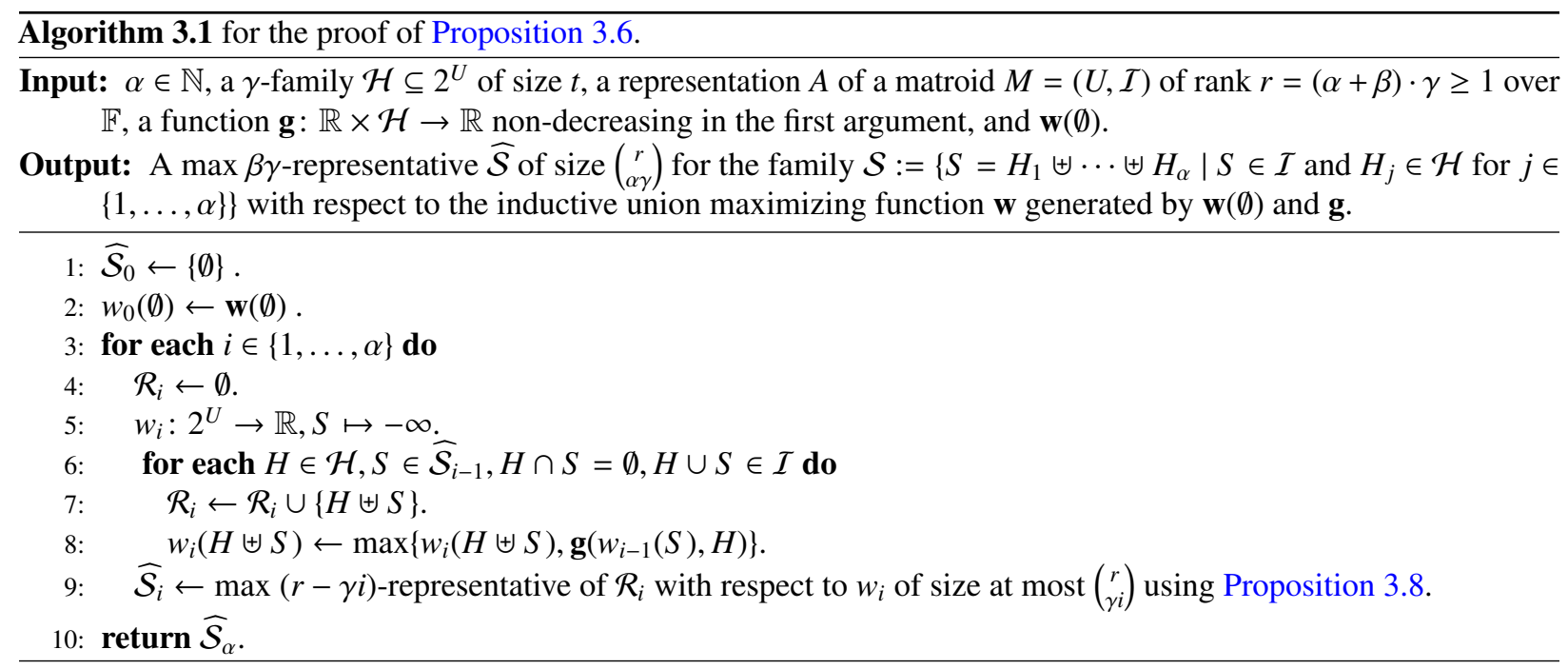

We now prove Proposition 3.6. Like its implicit ad-hoc proofs in the literature $[14,15,25]$, we will prove it by iterative application of the following known result.

Proposition 3.8 (Fomin et al. [14, Theorem 3.7]). Let $M=(U, \mathcal{I})$ be a linear matroid of rank $r=\alpha+\beta, \mathcal{S}=$ $\left\{S_{1}, \ldots, S_{t}\right\}$ be an $\alpha$-family of independent sets, and $w: \mathcal{S} \rightarrow \mathbb{R} .{ }^{1}$ Then, there exists a max $\beta$-representative $\widehat{\mathcal{S}} \subseteq \mathcal{S}$ of size at most $\left(\begin{array}{c}r \\ \alpha\end{array}\right)$. Given a representation of $M$ over a field $\mathbb{F}, \widehat{\mathcal{S}}$ can be found using

$$
O\left(\left(\begin{array}{l}
r \\
\alpha
\end{array}\right) t \alpha^{\omega}+t\left(\begin{array}{l}
r \\
\alpha
\end{array}\right)^{\omega-1}\right) \text { operations over } \mathbb{F},
$$

where $\omega$ is any constant such that two $(n \times n)$-matrices can be multiplied in $O\left(n^{\omega}\right)$ time.

Algorithm 3.1 computes the representative families required by Proposition 3.6 by iteratively applying Proposition 3.8. Proposition 3.6 follows from the correctness proof of Algorithm 3.1 in Lemma 3.9 and its running time analysis in Lemma 3.10.

Lemma 3.9. Algorithm 3.1 is correct.

Proof. We prove by induction that,

(i) in line 9 of Algorithm 3.1, $\widehat{\mathcal{S}}_{i}$ is $\max (r-\gamma i)$-representative with respect to $\mathbf{w}$ for

$$
\mathcal{S}_{i}=\left\{S=H_{1} \uplus \cdots \uplus H_{i} \mid S \in \mathcal{I} \text { and } H_{j} \in \mathcal{H} \text { for } j \in\{1, \ldots, i\}\right\} \text {. }
$$

(ii) To this end, we simultaneously show that $w_{i}(X)=\mathbf{w}(X)$ for $X \in \mathcal{R}_{i}$.

The lemma then follows since Algorithm 3.1 returns $\widehat{\mathcal{S}}_{\alpha}$ in line 10, which has size $\left(\begin{array}{c}r \\ \alpha \gamma\end{array}\right)$ by construction in line 9.

Both (i) and (ii) hold for $i=1$ since $\mathcal{S}_{1}=\mathcal{R}_{1}=\mathcal{H} \cap \mathcal{I}$ and $\mathbf{w}(X)=\mathbf{g}(\mathbf{w}(\emptyset), X)=w_{1}(X)$ for all $X \in \mathcal{H}$ by Definition 3.2. For the induction step, assume that (i) and (ii) hold for $i-1$ and observe that

(a) by construction, $\widehat{\mathcal{S}}_{i} \subseteq \mathcal{R}_{i} \subseteq \mathcal{S}_{i}$ for all $i \in\{1, \ldots, k\}$ and

\footnotetext{
${ }^{1}$ Fomin et al. [14] require the weight function to be non-negative. Yet their proof does not exploit non-negativity. Moreover, one can always transform the weight function so that it is non-negative and then transform it back.
} 
(b) since $\mathcal{H}$ is a $\gamma$-family and every subset of an independent set is independent, for all $X=H \uplus S \in \mathcal{S}_{i}$ with $H \in \mathcal{H}$ and $S \in \mathcal{B}(\mathcal{H})$, one has $S \in \mathcal{S}_{i-1}$.

We first prove (ii). For each set $X$ added to $\mathcal{R}_{i}$ by Algorithm 3.1 in line 7,

$$
w_{i}(X)=\max \left\{\mathbf{g}\left(w_{i-1}(S), H\right) \mid H \in \mathcal{H}, S \in \widehat{\mathcal{S}}_{i-1}, X=H \uplus S\right\} .
$$

Since, by induction, $w_{i-1}(X)=\mathbf{w}(X)$ for all $X \in \widehat{\mathcal{S}}_{i-1} \subseteq \mathcal{R}_{i-1}$, we have

$$
w_{i}(X)=\max \left\{\mathbf{g}(\mathbf{w}(S), H) \mid H \in \mathcal{H}, S \in \widehat{\mathcal{S}}_{i-1}, X=H \uplus S\right\} .
$$

Since, by induction, $\widehat{\mathcal{S}}_{i-1}$ is max representative for $\mathcal{S}_{i-1}$ with respect to $\mathbf{w}$, since $\mathbf{g}$ is non-decreasing in its first argument, and due to (b), we have

$$
\begin{aligned}
w_{i}(X) & =\max \left\{\mathbf{g}(\mathbf{w}(S), H) \mid H \in \mathcal{H}, S \in \mathcal{S}_{i-1}, X=H \uplus S\right\} \\
& =\max \{\mathbf{g}(\mathbf{w}(S), H) \mid H \in \mathcal{H}, S \in \mathcal{B}(\mathcal{H}), X=H \uplus S\}=\mathbf{w}(X),
\end{aligned}
$$

where the last equality is due to Definition 3.2.

We now show (i). In line 9, using Proposition 3.8, we create a max $(r-\gamma i)$-representative $\widehat{\mathcal{S}}_{i}$ of $\mathcal{R}_{i}$ with respect to $w_{i}$, which coincides with $\mathbf{w}$ on $\mathcal{R}_{i}$ by (ii). The claim now is that $\widehat{\mathcal{S}}_{i}$ is $\max (r-\gamma i)$-representative for $\mathcal{S}_{i}$ with respect to $\mathbf{w}$.

First, let $Y \subseteq U$ with $|Y|=r-\gamma i$ be such that there is an $X \in \mathcal{S}_{i}$ with $Y \uplus X \in \mathcal{I}$. Since $\mathbf{w}$ is an inductive union maximizing function (cf. Definition 3.2), there is a partition $S \uplus H=X$ with $H \in \mathcal{H}, S \in \mathcal{B}(\mathcal{H})$ such that

$$
\mathbf{w}(X)=\mathbf{g}(\mathbf{w}(S), H) .
$$

Since $X \in \mathcal{S}_{i}$, one has $S \in \mathcal{S}_{i-1}$ by (b). By induction, $\widehat{\mathcal{S}}_{i-1}$ is $\max (r-\gamma(i-1))$-representative for $\mathcal{S}_{i-1}$ with respect to w. Thus, there is a set $S^{\prime} \in \widehat{\mathcal{S}}_{i-1}$ with $(Y \uplus H) \uplus S^{\prime} \in \mathcal{I}$, and $\mathbf{w}\left(S^{\prime}\right) \geq \mathbf{w}(S)$. By construction of $\mathcal{R}_{i}$ in line $7, S^{\prime} \uplus H \in \mathcal{R}_{i}$. Since, by line $9, \widehat{\mathcal{S}}_{i}$ is $\max (r-\gamma i)$-representative for $\mathcal{R}_{i}$ with respect to $w_{i}$, there finally is an $X^{\prime} \in \widehat{\mathcal{S}}_{i}$ with $Y \uplus X^{\prime} \in \mathcal{I}$, and $\mathbf{w}\left(X^{\prime}\right)=w_{i}\left(X^{\prime}\right) \geq w_{i}\left(S^{\prime} \uplus H\right)=\mathbf{w}\left(S^{\prime} \uplus H\right)$. Since $\mathbf{g}$ is non-decreasing in its first argument and by (1), we get $\mathbf{w}\left(X^{\prime}\right) \geq \mathbf{w}\left(S^{\prime} \uplus H\right) \geq \mathbf{g}\left(\mathbf{w}\left(S^{\prime}\right), H\right) \geq \mathbf{g}(\mathbf{w}(S), H)=\mathbf{w}(X)$.

Finally, consider $Y \subseteq U$ with $|Y|<r-\gamma i$ such that there is an $X \in \mathcal{S}_{i}$ with $Y \uplus X \in \mathcal{I}$. Since matroid $M$ has rank $r$, there is a superset $Y^{\prime} \supseteq Y$ with $\left|Y^{\prime}\right|=r-\gamma i$ such that $Y^{\prime} \uplus X \in \mathcal{I}$. Thus, there exists $X^{\prime} \in \widehat{\mathcal{S}_{i}}$ such that $Y^{\prime} \uplus X^{\prime} \in \mathcal{I}$, and $\mathbf{w}\left(X^{\prime}\right) \geq \mathbf{w}(X)$ and both properties hold when replacing $Y^{\prime}$ by $Y$.

Having shown that Algorithm 3.1 is correct, to prove Proposition 3.6, it remains to show that Algorithm 3.1 runs in the claimed time.

Lemma 3.10. Algorithm 3.1 runs in time of $O\left(2^{\omega r} \cdot t\right)$ operations over $\mathbb{F}$ and calls to the function $\mathbf{g}$, where $\omega \geq 2$ is any constant such that two $(n \times n)$-matrices can be multiplied in $O\left(n^{\omega^{\prime}}\right)$ time for $\omega^{\prime}<\omega$.

Proof. Without loss of generality, $U=\{1, \ldots, n\}$. We represent subsets of $U$ as sorted lists. Since the input sets in $\mathcal{H}$ have cardinality $\gamma$, we can initially sort each of them in $O(\gamma \log \gamma)$ time. The sorted union and intersection of a sorted list of length $a$ and a sorted list of length $b$ can be computed in $O(a+b)$ time [3, Section 4.4]. We thus get a representation of sets as words of length $r$ over an alphabet of size $n$. We can thus store and look up the weight of a set of size at most $r$ in a trie in $O(r)$ time [3, Section 5.3]. Note that we do not have the time to completely initialize the $O(t)$ size- $n$ arrays in the trie nodes. Instead, we will initialize each array cell of a trie node at the first access: to keep track of the already initialized array positions, we use a data structure for sparse sets over a fixed universe $U$ that allows membership tests, insertion, and deletion of elements in constant time [7].

The running time of Algorithm 3.1 is dominated by the $\alpha \leq r$ iterations of the for-loop starting in line 3 . We analyze the running time of iteration $i$ of this loop. The family $\mathcal{H}$ consists of $t$ sets of size $\gamma$. The family $\widehat{\mathcal{S}}_{i-1}$ consists of $\left(\begin{array}{c}r \\ \gamma(i-1)\end{array}\right)$ sets of size $\gamma(i-1)$ by construction and Proposition 3.8. Thus, the for-loop starting in line 6 makes at most $2^{r} \cdot t$ iterations: 
(i) $H \cap S=\emptyset$ can be checked in $O(\gamma i) \subseteq O(r)$ time.

(ii) We check $H \uplus S \in \mathcal{I}$ by testing $|H \uplus S| \leq r$ columns of matrix $A$ of height $r$ for linear independence in time $O\left(r^{\omega^{\prime}}\right)[8]$.

(iii) The running time of lines 7 and 8 is dominated by looking up and storing weights of sets of size at most $r$ in $O(r)$ time using a trie, and a call to $\mathbf{g}$.

Thus, the for-loop in line 6 runs in time of $O\left(2^{r} r^{\omega^{\prime}} \cdot t\right)$ operations over $\mathbb{F}$ and calls to g. Finally, in line 9 , we build a $\max (r-\gamma i)$-representative of the $\gamma i$-family $\mathcal{R}_{i}$. Since $\left|\mathcal{R}_{i}\right| \leq 2^{r} \cdot t$, by Proposition 3.8, this works in time of

$$
O\left(\left(\left(\begin{array}{c}
r \\
\gamma i
\end{array}\right)(\gamma i)^{\omega^{\prime}}+\left(\begin{array}{c}
r \\
\gamma i
\end{array}\right)^{\omega^{\prime}-1}\right) \cdot\left|\mathcal{R}_{i}\right|\right) \subseteq O\left(\left(2^{r} r^{\omega^{\prime}}+2^{r\left(\omega^{\prime}-1\right)}\right) \cdot 2^{r} \cdot t\right) \subseteq O\left(\left(2^{2 r} r^{\omega^{\prime}}+2^{\omega^{\prime} r}\right) \cdot t\right) \subseteq O\left(2^{\omega^{\prime} r} r^{\omega^{\prime}} \cdot t\right)
$$

operations over $\mathbb{F}$, which dominates the running time of the for-loop in line 6. Thus, Algorithm 3.1 runs in time of $O\left(r \cdot 2^{\omega^{\prime} r} r^{\omega^{\prime}} \cdot t\right) \subseteq O\left(2^{\omega r} \cdot t\right)$ operations over $\mathbb{F}$ and calls to $\mathbf{g}$

\subsection{Computing intersection representative families}

In this section, we generalize Proposition 3.6 from representatives for a single matroid to matroid intersections.

Theorem 3.11. Let $\left\{M_{i}=\left(U, \mathcal{I}_{i}\right)\right\}_{i=1}^{m}$ be linear matroids of rank $r:=(\alpha+\beta) \gamma \geq 1, \mathcal{H} \subseteq 2^{U}$ be a $\gamma$-family of size $t$, and $\mathbf{w}: \mathcal{B}(\mathcal{H}) \rightarrow \mathbb{R}$ be an inductive union maximizing function (cf. Definition 3.2) generated by $\mathbf{w}(\emptyset)$ and the function $\mathbf{g}: \mathbb{R} \times \mathcal{H} \rightarrow \mathbb{R}$ non-decreasing in the first argument.

Given $\alpha \in \mathbb{N}$, a representation $A_{i}$ of $M_{i}$ for each $i \in\{1, \ldots, m\}$ over the same field $\mathbb{F}$, the value $\mathbf{w}(\emptyset)$, and the function $\mathbf{g}$, one can compute a max intersection $\beta \gamma$-representative of size at most $\left(\begin{array}{c}r m \\ \alpha \gamma m\end{array}\right)$ of the family

$$
\mathcal{S}=\left\{S=H_{1} \uplus \cdots \uplus H_{\alpha} \mid S \in \bigcap_{i=1}^{m} \mathcal{I}_{i} \text { and } H_{j} \in \mathcal{H} \text { for } j \in\{1, \ldots, \alpha\}\right\}
$$

with respect to $\mathbf{w}$ in time of $O\left(2^{\omega r m} \cdot(t+n)\right)$ operations over $\mathbb{F}$ and calls to the function $\mathbf{g}$, where $\omega \geq 2$ is any constant such that two $(n \times n)$-matrices can be multiplied in $O\left(n^{\omega^{\prime}}\right)$ time for $\omega^{\prime}<\omega$.

To prove Theorem 3.11, we reduce the $m$ matroid constraints to a single matroid constraint. To this end, we use a folklore construction sketched by Lawler [21, page 359] in a reduction of the Matroid INTERSECTION to the MatroID PARITY problem. It works at the expense of replacing each universe element by a "block" of $m$ copies that is only allowed to be completely included in or excluded from an independent set. We then use our Proposition 3.6 to compute a representative of the family of independent sets that can be obtained as unions of these "blocks". We now present the folklore construction and then prove Theorem 3.11 .

Lemma 3.12. Let $\left\{M_{i}=\left(U, \mathcal{I}_{i}\right)\right\}_{i=1}^{m}$ be linear matroids of rank $r$ and $\mathcal{H} \subseteq 2^{U}$,

$$
\begin{aligned}
U_{\oplus} & :=\left\{u^{(1)}, \ldots, u^{(m)} \mid u \in U\right\}, \text { and } \\
f: 2^{U} \rightarrow 2^{U_{\oplus}}, X & \mapsto\left\{u^{(1)}, \ldots, u^{(m)} \mid u \in X\right\} .
\end{aligned}
$$

Then, for all $S, S^{\prime} \subseteq U$,

(i) $S \neq S^{\prime} \Longleftrightarrow f(S) \neq f\left(S^{\prime}\right)$, that is, $f$ is injective,

(ii) $f(S) \cup f\left(S^{\prime}\right)=f\left(S \cup S^{\prime}\right)$,

(iii) $S \cap S^{\prime}=\emptyset \Longleftrightarrow f(S) \cap f\left(S^{\prime}\right)=\emptyset$,

and given $(r \times n)$-representations $A_{i}$ of $M_{i}$ for all $i \in\{1, \ldots, m\}$ over the same field $\mathbb{F}$, one can, in time of $O\left(m^{2} \cdot r\right.$. $n$ ) operations over $\mathbb{F}$, compute a $(\mathrm{rm} \times \mathrm{nm})$-representation $A_{\oplus}$ of a matroid $M_{\oplus}=\left(U_{\oplus}, I_{\oplus}\right)$ over $\mathbb{F}$ such that 


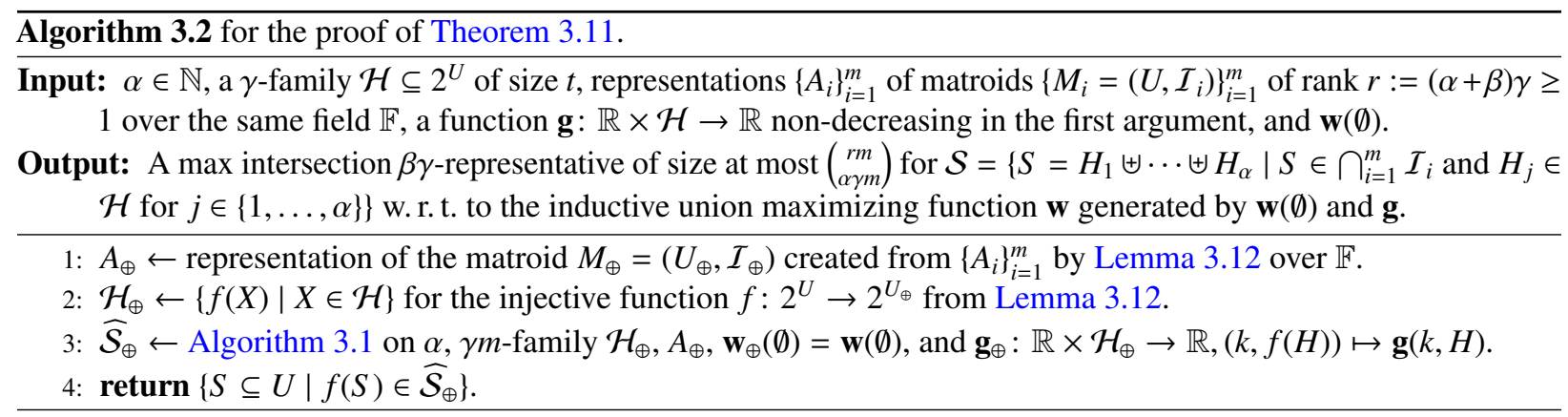

(iv) for all $S \subseteq U, S \in \bigcap_{i=1}^{m} \mathcal{I}_{i} \Longleftrightarrow f(S) \in \mathcal{I}_{\oplus}$.

Proof. We choose $M_{\oplus}$ to be the direct sum of pairwise disjoint copies $M_{i}^{\prime}$ of $M_{i}$ :

$$
\begin{aligned}
& M_{\oplus}=\left(U_{\oplus}, \mathcal{I}_{\oplus}\right)=\bigoplus_{i=1}^{m} M_{i}^{\prime} \text {, where } M_{i}^{\prime}=\left(U_{i}, \mathcal{I}_{i}^{\prime}\right) \text { with } \\
& U_{i}:=\left\{u^{(i)} \mid u \in U\right\} \text { and } \mathcal{I}_{i}^{\prime}:=\left\{\left\{u_{1}^{(i)}, \ldots, u_{j}^{(i)}\right\} \mid\left\{u_{1}, \ldots, u_{j}\right\} \in \mathcal{I}_{i}\right\} .
\end{aligned}
$$

We get a $(r m \times n m)$-representation $A_{\oplus}$ over $\mathbb{F}$ of $M_{\oplus}$ in time of $O\left(m^{2} \cdot r \cdot n\right)$ operations over $\mathbb{F}$ [26, Exercise 6, p. 132].

Properties (i)-(iii) obviously hold by construction. It remains to prove (iv). Let $S=\left\{u_{1}, \ldots, u_{j}\right\} \subseteq U$ and, for an arbitrary $i \in\{1, \ldots, m\}$, let $S_{i}=f(S) \cap U_{i}$. Then, $S_{i}=\left\{u_{1}^{(i)}, \ldots, u_{j}^{(i)}\right\}$ and $S \in \mathcal{I}_{i}$ if and only if $S_{i} \in \mathcal{I}_{i}^{\prime}$. Thus, if $S \in \bigcap_{i=1}^{m} \mathcal{I}_{i}$, then $S_{i} \in \mathcal{I}_{i}^{\prime}$ for all $i \in\{1, \ldots, m\}$ and, hence, $\bigcup_{i=1}^{m} S_{i}=f(S) \in \mathcal{I}_{\oplus}$ by Proposition 2.8 on direct sums. Conversely, if $f(S) \in \mathcal{I}_{\oplus}$, then $S_{i} \in \mathcal{I}_{i}^{\prime}$ for all $i \in\{1, \ldots, m\}$ and, therefore, $S \in \bigcap_{i=1}^{m} \mathcal{I}_{i}$.

Theorem 3.11 now follows from the following lemma.

Lemma 3.13. Algorithm 3.2 is correct and runs in time of $O\left(2^{\omega r m} \cdot(t+n)\right)$ operations over $\mathbb{F}$ and calls to $\mathbf{g}$. If $A_{\oplus}$ in line 1 is given (for example, precomputed), then Algorithm 3.2 runs in time of $O\left(2^{\omega r m} \cdot t\right)$ operations over $\mathbb{F}$ and calls to $\mathbf{g}$, where $\omega \geq 2$ is any constant such that two $(n \times n)$-matrices can be multiplied in $O\left(n^{\omega^{\prime}}\right)$ time for $\omega^{\prime}<\omega$.

Proof. In line 1, from the linear representations $\left\{A_{i}\right\}_{i=1}^{m}$ of the matroids $\left\{M_{i}\right\}_{i=1}^{m}$, Algorithm 3.2 computes a $(\mathrm{rm} \times \mathrm{nm})$ representation $A_{\oplus}$ of the matroid $M_{\oplus}=\left(U_{\oplus}, \mathcal{I}_{\oplus}\right)$ of rank $r m=(\alpha+\beta) \gamma m$ from Lemma 3.12 in time of $O\left(m^{2}\right.$. $r \cdot n$ ) operations over $\mathbb{F}$. In line 2 , it computes the $\gamma m$-family $\mathcal{H}_{\oplus}$. By Lemma 3.9, the result of line 3 is a max $\beta \gamma m$-representative $\widehat{\mathcal{S}}_{\oplus}$ of size $\left(\begin{array}{c}r m \\ \alpha \gamma m\end{array}\right)$ for

$$
\mathcal{S}_{\oplus}:=\{f(S) \mid S \in \mathcal{S}\}=\left\{f(S)=f\left(H_{1}\right) \uplus \cdots \uplus f\left(H_{\alpha}\right) \mid f\left(H_{i}\right) \in \mathcal{H}_{\oplus} \text { and } f(S) \in \mathcal{I}_{\oplus}\right\},
$$

where equality is due to Lemma 3.12(ii) and (iv), with respect to the inductive union maximizing function $\mathbf{w}_{\oplus}$ determined by $\mathbf{w}_{\oplus}(\emptyset)=\mathbf{w}(\emptyset)$ and the non-decreasing in its first argument function $\mathbf{g}_{\oplus}$. By Lemma 3.10, line 3 is executed in time of $O\left(2^{\omega r m} \cdot t\right)$ operations over $\mathbb{F}$ and calls to $\mathbf{g}_{\oplus}$. Hence, together with applying the transformation from Lemma 3.12 , we take the time of $O\left(2^{\omega r m} \cdot(t+n)\right)$ operations over $\mathbb{F}$ and calls to $\mathbf{g}_{\oplus}$. Herein, one call to $\mathbf{g}_{\oplus}$ is one call to $\mathbf{g}$. Also, since $\mathbf{g}_{\oplus}(k, f(H))=\mathbf{g}(k, H)$ for all $k \in \mathbb{R}$ and all $f(H) \in \mathcal{H}_{\oplus}$, one has $\mathbf{w}_{\oplus}(f(X))=\mathbf{w}(X)$ for all $f(X) \in \mathcal{B}\left(\mathcal{H}_{\oplus}\right)$. This allows us to show that the result returned in line 4 ,

$$
\widehat{\mathcal{S}}:=\left\{S \subseteq U \mid f(S) \in \widehat{\mathcal{S}}_{\oplus}\right\}=\left\{H_{1} \uplus \cdots \uplus H_{\alpha} \mid H_{i} \in \mathcal{H} \text { and } \biguplus_{i=1}^{\alpha} f\left(H_{i}\right) \in \widehat{\mathcal{S}}_{\oplus}\right\},
$$

which has size $\left|\widehat{\mathcal{S}}_{\oplus}\right|$, is $\max \beta \gamma$-intersection representative of $\mathcal{S}$ with respect to w. Note that $\widehat{\mathcal{S}}$ can be constructed in $\alpha \gamma m \cdot\left|\widehat{\mathcal{S}}_{\oplus}\right|$ time by simply iterating over the sets in $\widehat{\mathcal{S}}_{\oplus}$, replacing a group of elements $u^{(1)}, \ldots, u^{(m)}$ by element $u$. 
To see that $\widehat{\mathcal{S}}$ is $\max \beta \gamma$-intersection representative of $\mathcal{S}$ with respect to $\mathbf{w}$, consider set $Y \subseteq U$ with $|Y| \leq \beta \gamma$ and $X \in \mathcal{S}$ with $Y \uplus X \in \bigcap_{i=1}^{m} \mathcal{I}_{i}$. Then $f(Y) \uplus f(X) \in \mathcal{I}_{\oplus}$ by Lemma 3.12(iii) and (iv). Moreover, $f(X) \in \mathcal{S}_{\oplus}$. By Definition 3.1, there is a set $X^{\prime} \in \widehat{\mathcal{S}}_{\oplus}$ such that $f(Y) \uplus X^{\prime} \in \mathcal{I}_{\oplus}$, and $\mathbf{w}_{\oplus}\left(X^{\prime}\right) \geq \mathbf{w}_{\oplus}(f(X))$. By construction of $\widehat{\mathcal{S}}$, there is an $X^{\prime \prime} \in \widehat{\mathcal{S}}$ with $f\left(X^{\prime \prime}\right)=X^{\prime}$. Note that $Y \cap X^{\prime \prime}=\emptyset$ by Lemma 3.12(iii) since $f(Y) \cap f\left(X^{\prime \prime}\right)=f(Y) \cap X^{\prime}=\emptyset$. Moreover, $Y \cup X^{\prime \prime} \in \bigcap_{i=1}^{m} I_{i}$ by Lemma 3.12(iv) since $f(Y) \uplus f\left(X^{\prime \prime}\right)=f(Y) \uplus X^{\prime} \in \mathcal{I}_{\oplus}$. Finally, $\mathbf{w}\left(X^{\prime \prime}\right)=\mathbf{w}_{\oplus}\left(f\left(X^{\prime \prime}\right)\right)=\mathbf{w}_{\oplus}\left(X^{\prime}\right) \geq$ $\mathbf{w}_{\oplus}(f(X))=\mathbf{w}(X)$.

\section{Set packing with multiple matroid constraints}

In this section, we apply the results from Section 3 to obtain a fixed-parameter algorithm for the following problem.

Problem 4.1 (Set Packing with Matroid Constraints (SPMC)).

Input: Matroids $\left\{\left(U, \mathcal{I}_{i}\right)\right\}_{i=1}^{m}$, a family $\mathcal{H} \subseteq 2^{U}, w: \mathcal{H} \rightarrow \mathbb{R}$, and $\alpha \in \mathbb{N}$.

Task: Find sets $H_{1}, \ldots, H_{\alpha} \in \mathcal{H}$ such that

$$
\biguplus_{i=1}^{\alpha} H_{i} \in \bigcap_{i=1}^{m} \mathcal{I}_{i} \quad \text { maximizing } \quad \sum_{i=1}^{\alpha} w\left(H_{i}\right) .
$$

SPMC is a generalization of the Matroid Parity and Matroid Matching problems introduced by Lawler [21] as generalization of the 2-Matroid Intersection problem. In Matroid Parity and Matroid Matching, there is only one input matroid and all input sets in $\mathcal{H}$ have size exactly two. In MATroID PARITY, all input sets are additionally required to be pairwise disjoint. Both problems are solvable in polynomial-time on linear matroids, but not on general matroids [28, Section 43.9]. Lee et al. [22] studied approximation algorithms for the variant Matroid HyPergraPH Matching with one input matroid and unweighted (but possibly intersecting) input sets. Finally, Marx [25] and Lokshtanov et al. [23] obtained fixed-parameter tractability results for MATROID $\gamma$-PARITY, in which only one matroid is given in the input and the input set family consists of pairwise non-intersecting unweighted sets of size $\gamma$. We generalize the fixed-parameter algorithms of Marx [25] and Lokshtanov et al. [23] to SPMC.

Theorem 4.2. SPMC with sets of size at most $\gamma$ and $m$ matroids of rank at most $r$ with given representations over a field $\mathbb{F}=\mathbb{F}_{p^{d}}$ is solvable in time of $2^{O(\alpha \gamma m)} \cdot|\mathcal{H}|^{2} \cdot \operatorname{poly}(r)+m^{2} n \cdot \operatorname{poly}(r, \alpha, \gamma, p, d)$ operations over $\mathbb{F}$.

Proof. We will prove the theorem using Algorithm 4.1, which computes weight of an optimal solution to SPMC. The actual solution can then be retrieved via self-reduction, calling Algorithm 4.1 as most $|\mathcal{H}|$ times. However, note that for the repeated application of Algorithm 4.1, it is enough to compute the matroid representations $\left\{A_{i}^{*}\right\}_{i=1}^{m},\left\{A_{i}^{\prime}\right\}_{i=1}^{m}$, and $A_{\oplus}$ in Algorithm 3.2 called in line 8 once, as they do not depend on $\mathcal{H}$. Thus, we will only once account for the time of their computation and analyze the running time of $|\mathcal{H}|$ calls of Algorithm 4.1 under the assumption that they are precomputed.

First, in lines 1 to 4 , Algorithm 4.1 constructs a family $\mathcal{H}^{\prime}$ from $\mathcal{H}$ in which each set has size exactly $\gamma$. This step can be executed in $|\mathcal{H}| \alpha \gamma$ time. In line 6, for each $i \in\{1, \ldots, m\}$ and $r_{i}$ being the rank of $M_{i}=\left(U, \mathcal{I}_{i}\right)$, we compute a representation $A_{i}^{*}$ of matroid $M_{i}^{*}=\left(U^{*}, \mathcal{I}_{i}^{*}\right)$ of rank $r_{i}+|D|=r_{i}+\alpha \gamma$ in $O\left(\left(r_{i}+\alpha \gamma\right)(n+\alpha \gamma)\right)$ operations over $\mathbb{F}$ by Lemma 2.11. Note that $\left|U^{*}\right|=n+|D|=n+\alpha \gamma$ and

$$
\begin{array}{rr}
\text { there are sets } H_{1}, \ldots, H_{\alpha} \in \mathcal{H} & \text { with } \biguplus_{i=1}^{\alpha} H_{i} \in \bigcap_{i=1}^{m} \mathcal{I}_{i} \quad \text { if and only if } \\
\text { there are sets } H_{1}^{(1)}, \ldots, H_{\alpha}^{(\alpha)} \in \mathcal{H}^{\prime} & \text { with } \biguplus_{i=1}^{\alpha} H_{i}^{(i)} \in \bigcap_{i=1}^{m} \mathcal{I}_{i}^{*} .
\end{array}
$$

In line 7, we compute $\alpha \gamma$-truncations $\left\{A_{i}^{\prime}\right\}_{i=1}^{m}$ of the $\left\{A_{i}^{*}\right\}_{i=1}^{m}$ using Corollary 2.15 in time of

$$
\begin{array}{r}
O(m \cdot(n+\alpha \gamma) \cdot \alpha \gamma \cdot(r+\alpha \gamma))+(r+\alpha \gamma)^{4} \cdot(\alpha \gamma)^{4} \cdot d^{2} \sqrt{p} \cdot \operatorname{polylog}(r+\alpha \gamma, p, d) \\
\subseteq m n \cdot \operatorname{poly}(r, \alpha, \gamma, p, d) \text { operations over } \mathbb{F}
\end{array}
$$




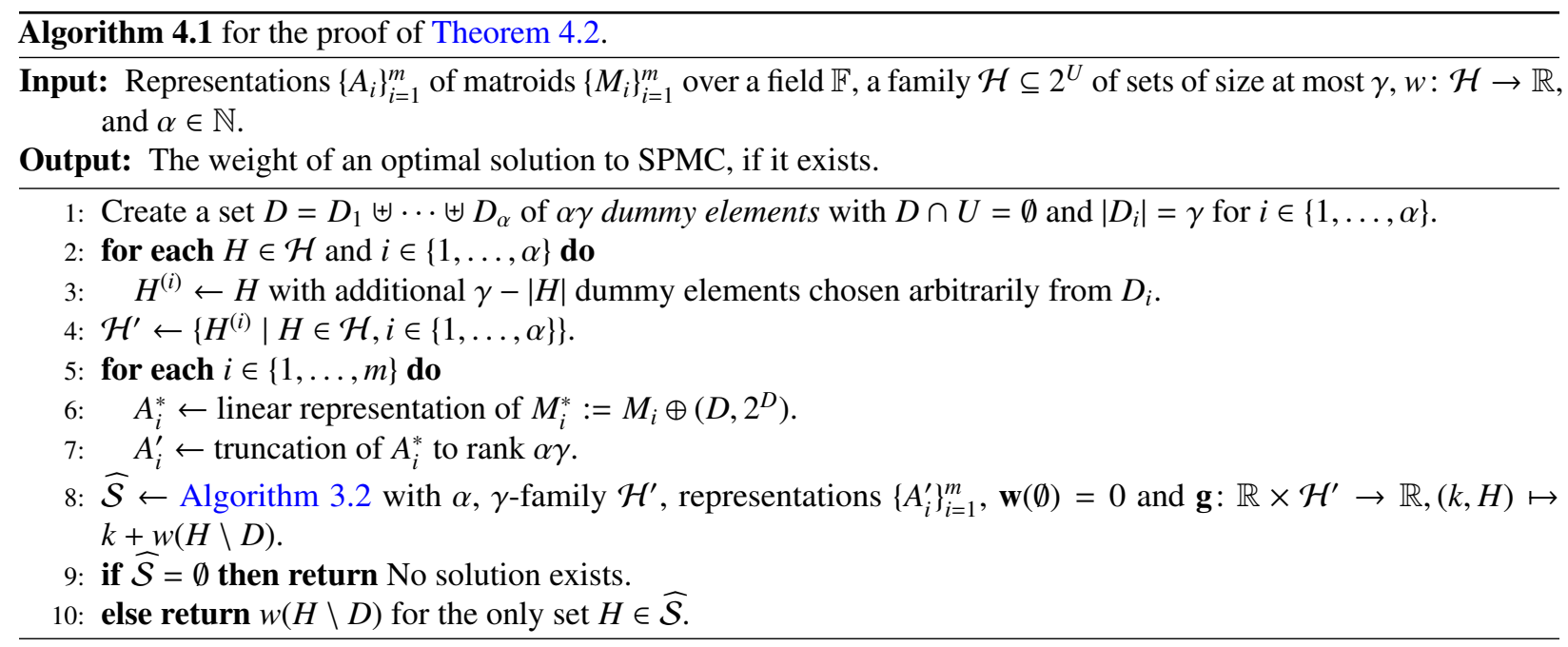

and obtain an $(\alpha \gamma \times(n+\alpha \gamma))$-representation $A_{i}^{\prime}$ of $M_{i}^{\prime}$ over $\mathbb{F}^{\prime}:=\mathbb{F}_{p^{(r+\alpha \gamma) \alpha \gamma d}}$ for each $i \in\{1, \ldots, m\}$. Note that any operation over $\mathbb{F}^{\prime}$ can be executed using poly $(r, \alpha, \gamma)$ operations over $\mathbb{F}$.

In line 8, we apply Algorithm 3.2 to the linear representations $\left\{A_{i}^{\prime}\right\}_{i=1}^{m}$ of the matroids $\left\{M_{i}^{\prime}\right\}_{i=1}^{m}$, all of rank $\alpha \gamma$. By Lemma 3.13, the result will be a max 0 -representative $\widehat{\mathcal{S}}$ of size $\left(\begin{array}{c}\alpha \gamma m \\ \alpha \gamma m\end{array}\right)=1$ of the family

$$
\mathcal{S}:=\left\{S=H_{1} \uplus \cdots \uplus H_{\alpha} \mid S \in \bigcap_{i=1}^{m} \mathcal{I}_{i}^{\prime} \text { and } H_{i} \in \mathcal{H}^{\prime} \text { for } i \in\{1, \ldots, \alpha\}\right\}
$$

with respect to the inductive union maximizing function determined by $\mathbf{w}(\emptyset)=0$ and $\mathbf{g}: \mathbb{R} \times \mathcal{H}^{\prime} \rightarrow \mathbb{R},(k, H) \mapsto$ $k+w(H \backslash D)$. By Lemma 3.13, this takes time of $2^{O(\alpha \gamma m)} \cdot|\mathcal{H}|$ operations over $\mathbb{F}^{\prime}$ and calls to $\mathbf{g}$ if the $A_{\oplus}$ in Algorithm 3.2 is precomputed. The overall running time of solving SPMC is thus $|\mathcal{H}|$ applications of line 8 in $2^{O(\alpha \gamma m)} \cdot \operatorname{poly}(r) \cdot|\mathcal{H}|^{2}$ operations over $\mathbb{F}$ and calls to $\mathbf{g}$, plus $m^{2} n \cdot \operatorname{poly}(r, \alpha, \gamma, p, d)$ operations over $\mathbb{F}$ for computing $A_{\oplus}$ from Lemma 3.12 , the $\left\{A_{i}^{*}\right\}_{i=1}^{m}$, and the $\left\{A_{i}^{\prime}\right\}_{i=1}^{m}$ once.

We finally prove that line 10 returns the weight of an optimal solution to our input SPMC instance if and only if that instance has a feasible solution. The weight function on $\mathcal{S}$ generated by $\mathbf{w}(\emptyset)=0$ and $\mathbf{g}$ is

$$
w_{\Sigma}(X):=\max _{\substack{X=H_{1} \uplus \cdots \uplus H_{\alpha} \\ H_{1}, \ldots, H_{\alpha} \in \mathcal{H}^{\prime}}} \sum_{i=1}^{\alpha} w^{\prime}\left(H_{i}\right), \quad \text { with } \quad w^{\prime}\left(H_{i}\right):=w\left(H_{i} \backslash D\right) .
$$

$(\geq)$ Let $S^{*}:=\biguplus_{i=1}^{\alpha} H_{i} \in \bigcap_{i=1}^{m} \mathcal{I}_{i}$ be an optimal solution to SPMC. One has $S:=\biguplus_{i=1}^{\alpha} H_{i}^{(i)} \in \bigcap_{i=1}^{m} \mathcal{I}_{i}^{*}$ by (2). Since $|S|=\alpha \gamma, S \in \bigcap_{i=1}^{m} \mathcal{I}_{i}^{\prime}$. Thus, $S \in \mathcal{S}$. Since $\widehat{\mathcal{S}}$ is max 0-representative, there is an $S^{\prime} \in \widehat{\mathcal{S}}$ with

$$
w_{\Sigma}\left(S^{\prime}\right) \geq w_{\Sigma}(S) \geq \sum_{i=1}^{\alpha} w^{\prime}\left(H_{i}^{(i)}\right)=\sum_{i=1}^{\alpha} w\left(H_{i}\right)
$$

( $\leq$ ) For $S \in \widehat{\mathcal{S}}$, there are $H_{1}^{(1)}, \ldots, H_{\alpha}^{(\alpha)} \in \mathcal{H}^{\prime}$ such that $\biguplus_{i=1}^{\alpha} H_{i}^{(i)} \in \bigcap_{i=1}^{m} \mathcal{I}_{i}^{\prime}$ and

$$
w_{\Sigma}(S)=\sum_{i=1}^{\alpha} w^{\prime}\left(H_{i}^{(i)}\right)=\sum_{i=1}^{\alpha} w\left(H_{i}\right)
$$

which is at most the weight of an optimal solution to SPMC, because the $H_{i}$ are a feasible solution: $\biguplus_{i=1}^{\alpha} H_{i}^{(i)} \in \bigcap_{i=1}^{m} \mathcal{I}_{i}^{*}$. Thus, by (2), $\biguplus_{i=1}^{\alpha} H_{i} \in \bigcap_{i=1}^{m} \mathcal{I}_{i}$. 


\section{Facility location with multiple matroid constraints}

Facility location problems are a classical topic studied in operations research [20]: each facility $u$ has an opening cost $c_{u}$, serving client $v$ by facility $u$ costs $p_{u v}$, and the task is to decide which facilities to open in order to minimize the total cost of serving all clients. Fellows and Fernau [12] show that this problem is fixed-parameter tractable when parameterized by an upper bound on the optimum solution cost, yet W[2]-hard when parameterized by the number of facilities that may be opened. Krishnaswamy et al. [19] and Swamy [30] study approximation algorithms for the problem variant where the set of facilities is required to be independent in a single matroid and show several applications. Kalhan [17] additionally studies capacity constraints on facilities.

We study facility location problems where not all clients have to be served, but where both the set of clients and the set of facilities are required to be independent in multiple matroids. In this case, the minimization problem is meaningless: it would be optimal not serve any clients and not open any facilities. Thus, we study the problem of maximizing income minus facility opening costs.

\section{Problem 5.1 (Uncapacitated facility location with matroid constraints (UFLP-MC)).}

Input: A universe $U$ with $n:=|U|$, for each pair $u, v \in U$ a profit $p_{u v} \in \mathbb{N}$ obtained when a facility at $u$ serves a client at $v$, for each $u \in U$ a cost $c_{u} \in \mathbb{N}$ for opening a facility at $u$, facility matroids $\left\{\left(U_{i}, A_{i}\right)\right\}_{i=1}^{a}$, and client matroids $\left\{\left(V_{i}, C_{i}\right)\right\}_{i=1}^{c}$, where $U_{i} \cup V_{i} \subseteq U$.

Task: Find two disjoint sets $A \uplus C \subseteq U$ that maximize the profit

$$
\sum_{v \in C} \max _{u \in A} p_{u v}-\sum_{u \in A} c_{u} \quad \text { such that } \quad A \in \bigcap_{i=1}^{a} A_{i} \quad \text { and } \quad C \in \bigcap_{i=1}^{c} C_{i} .
$$

By convention, the intersection of no sets is the whole universe. Thus, if $a=0$ or $c=0$, this is the same as giving matroids in which any set of facilities or clients is feasible. For UFLP-MC without matroid constraints, Ageev and Sviridenko [2] showed a 0.828-approximation algorithm and that there is no polynomial-time approximation scheme.

UFLP-MC with multiple matroid constraints can model natural facility location scenarios like "open at most $k_{i}$ facilities of type $i$ " [10], even if types are not disjoint, moving facilities [30], moving clients, yet can also model problems in social network analysis [5, Section 3]. It also generalizes fundamental covering problems:

Example 5.2. Using UFLP-MC with $a=1$ facility matroid and $c=0$ client matroids, one can model the classical $N P$-hard SET Cover problem [18] of covering a maximum number of elements of a set $V$ using at most $r$ sets of a collection $\mathcal{H} \subseteq 2^{V}$. To this end, choose the universe $U=V \cup \mathcal{H}$, a single facility matroid $\left(\mathcal{H}, A_{1}\right)$ with $A_{1}:=\{H \subseteq \mathcal{H}|| H \mid \leq r\}, c_{u}=0$ for each $u \in U$, and, for each $u, v \in U$,

$$
p_{u v}= \begin{cases}1 & \text { if } u \in \mathcal{H} \text { such that } v \in u, \\ 0 & \text { otherwise. }\end{cases}
$$

From Example 5.2 and the W[2]-hardness of SET Cover [11], it immediately follows that UFLP-MC is W[2]-hard parameterized by $r$ even for zero costs, binary profits, and a single uniform facility matroid. Hence, when the set of clients is unconstrained, the problem of optimally placing a small number $r$ of facilities is hard. However, facility location problems have also been studied with a small number of clients [1] and occur in several plausible scenarios [5]. We use the tools developed in Sections 3 and 4 to analyze the parameterized complexity of UFLP-MC with a small number of clients.

\section{Theorem 5.3. UFLP-MC is}

(i) W[1]-hard parameterized by $r$ for a single client matroid of rank $r$, even with unit costs, binary profits, and without facility matroids,

(ii) solvable in $2^{O(r \log r)} \cdot n^{2}$ time for a single uniform client matroid of rank $r$ and any a single facility matroid given as an independence oracle, 
(iii) fixed-parameter tractable parameterized by $a+c+r$, where $r$ is the minimum rank of the client matroids and representations of all matroids over the same finite field $\mathbb{F}_{p^{d}}$ are given, where $p$ is a prime polynomially upper-bounded by the input size.

Before proving Theorem 5.3, we put it into context. Theorem 5.3 generalizes and unifies several fixed-parameter tractability results from the literature. Marx [25] showed that a common independent set of size $r$ in $m$ matroids can be found in $f(r, m) \cdot \operatorname{poly}(n)$ time. Our Theorem 5.3(iii) is a direct generalization of this result. Bonnet et al. [6] showed that the problem of covering at least $p$ elements of a set $V$ using at most $k$ sets of a given family $\mathcal{H} \subseteq 2^{V}$ is fixed-parameter tractable parameterized by $p$. This result also follows from our Theorem 5.3(ii) using Example 5.2 with an additional uniform client matroid of rank $p$. Earlier, Marx [24] showed that Partial Vertex Cover (can one cover at least $p$ edges of a graph by at most $k$ vertices?) is fixed-parameter tractable by $p$. Our Theorem 5.3(ii) generalizes this result and, indeed, is based on the color coding approach in Marx's [24] algorithm.

We now prove Theorem 5.3: (i) is proved in Section 5.1, whereas (ii) and (iii) are proved in Section 5.2.

\subsection{W[1]-hardness for general client matroids (proof of Theorem 5.3(i))}

To prove Theorem 5.3(i), we exploit that the CulQue problem is W[1]-hard parameterized by $k$ [11]:

\section{Problem 5.4 (Clique).}

Input: A graph $G=(V, E)$ and integer $k \in \mathbb{N}$.

Question: Does $G$ contain a clique on $k$ vertices?

To transfer the W[1]-hardness of CLIQUE parameterized by $k$ to UFLP-MC parameterized by the client matroid rank, we reduce the problem of finding a clique of size $k$ to UFLP-MC with a client matroid of rank $2 k$. The reduction is inspired by the proof that MATROID PARITY is generally not polynomial-time solvable when matroids are given using an independence oracle [28, Section 43.9].

Construction 5.5. Let $(G, k)$ with $G=(V, E)$ be an instance of CLIQue. We construct an instance of UFLP-MC as follows. For each vertex $u \in V$, let $D_{u}:=\left\{u^{\prime}, u^{\prime \prime}\right\}$ such that $D_{u} \cap V=\emptyset$, let $L:=\left\{D_{u} \mid u \in V\right\}$, and $S:=\bigcup_{u \in V} D_{u}$. Our $U F L P$-MC instance consists of the universe $U=V \uplus S$, and, for all $u, v \in U$,

$$
c_{u}=\left\{\begin{array}{ll}
1, & \text { if } u \in V, \\
0, & \text { otherwise, }
\end{array} \quad p_{u v}= \begin{cases}1, & \text { if } u \in V \text { and } v \in D_{u}, \\
0, & \text { otherwise. }\end{cases}\right.
$$

We do not use a facility matroid. As client matroid, we use the known matroid $\left(S, I_{C}\right)$ [28, Section 43.9] with

$$
\begin{aligned}
\mathcal{I}_{C} & =\{J \subseteq S|| J \mid \leq 2 k-1\} \\
& \cup\{J \subseteq S|| J \mid=2 k \text { and } J \text { is not the union of any } k \text { pairs in } L\} \\
& \cup\left\{J \subseteq S|A \subseteq V,| A \mid=k, J=\biguplus_{u \in A} D_{u} \text {, and } G[A] \text { is a clique }\right\} .
\end{aligned}
$$

Proof (of Theorem 5.3(i)). Construction 5.5 works in polynomial time and creates an UFLP-MC instance with unit costs and binary profits as claimed in Theorem 5.3(i). Moreover, the rank of the client matroid is $2 k$. We now show that there is a clique of size $k$ in $G=(V, E)$ if and only if there is a solution to the created UFLP-MC instance with profit $k$.

$(\Rightarrow)$ Let $A \subseteq V,|A|=k, G[A]$ be a clique, and $C:=\biguplus_{u \in A} D_{u}$. Since $|C|=2 k$, it follows that $C \in \mathcal{I}_{C}$. Hence, $A \uplus C$ is a feasible solution to UFLP-MC. Since each $u \in A$ has $c_{u}=1$ and each $v \in C$ has $p_{u v}=1$ for the $u \in A$ with $v \in D_{u}$, the profit of $A \uplus C$ given by (4) is

$$
\sum_{v \in C} \max _{u \in A} p_{u v}-\sum_{u \in A} c_{u}=2 k-k=k .
$$

$(\Leftarrow)$ Let $A \uplus C$ be an inclusion-minimal solution with profit at least $k$ to the created UFLP-MC instance. Since each facility $u \in A$ has $c_{u}=1$ and since $p_{u v}=1$ if and only if $v \in D_{u} \cap C$, the profit of $A \uplus C$ given by (4) is

$$
-\sum_{u \in A} c_{u}+\sum_{v \in C} \max _{u \in A} p_{u v}=-|A|+\sum_{u \in A}\left|D_{u} \cap C\right| \geq k .
$$


Hence, if there is an $u \in A$ with $\left|D_{u} \cap C\right| \leq 1$, then $(A \backslash\{u\}) \uplus\left(C \backslash D_{u}\right)$ is a solution with at least the same profit, contradicting the minimality of $A \uplus C$. Thus, for each $u \in A$, we have $D_{u} \subseteq C$. Moreover, $|C| \leq 2 k$ since $C \in \mathcal{I}_{C}$ and the client matroid $\left(S, I_{C}\right)$ has rank $2 k$. Combining this with (6), we thus get

$$
2 k \geq \sum_{u \in A}\left|D_{u} \cap C\right|=2|A| \geq k+|A|,
$$

which means $|A|=k$. Thus, $C=\biguplus_{u \in A} D_{u},|C|=2 k, C \in \mathcal{I}_{C}$, and we conclude that $G[A]$ is a clique of size $k$.

\subsection{Fixed-parameter algorithms for linear client matroids (proof of Theorem 5.3(ii) and (iii))}

One major difficulty in solving UFLP-MC is that the profit from opening a facility depends on which facilities are already open. To name an extreme example: when opening only facility $u$, it induces cost $c_{u}$ and yields profit from serving all the clients. However, when some other facility $v$ is already open, then additionally opening $u$ induces cost $c_{u}$ yet might not yield any profit if all clients are more profitably already served by $v$. To avoid such interference between facilities, we reduce UFLP-MC to problem variant with matroid and color constraints (UFLP-MCC).

Problem 5.6 (UFLP-MCC).

Input: A universe $U$, a coloring col: $U \rightarrow\{1, \ldots, k+\ell\}$, a partition $Z_{1} \uplus \cdots \uplus Z_{\ell}=\{\ell+1, \ldots, \ell+k\}$, for each pair $u, v \in U$ a profit $p_{u v} \in \mathbb{N}$ gained when a facility at u serves a client at $v$, for each $u \in U$ a cost $c_{u} \in \mathbb{N}$ for opening a facility at $u$, facility matroids $\left\{\left(U_{i}, A_{i}\right)\right\}_{i=1}^{a}$, and client matroids $\left\{\left(V_{i}, C_{i}\right)\right\}_{i=1}^{c}$, where $U_{i} \cup V_{i} \subseteq U$.

Task: Find two sets $A \uplus C \subseteq U$ such that

(i) for each $i \in\{1, \ldots, \ell\}$, there is exactly one facility $u \in A$ with $\operatorname{col}(u)=i$,

(ii) for each $i \in\{\ell+1, \ldots, \ell+k\}$, there is exactly one client $v \in C$ with $\operatorname{col}(v)=i$,

(iii) $A \in \bigcap_{i=1}^{a} A_{i}$ and $C \in \bigcap_{i=1}^{c} C_{i}$,

and that maximizes

$$
\sum_{u \in A}\left(-c_{u}+\sum_{v \in C \cap Z(u)} p_{u v}\right), \text { where } Z(u):=\left\{v \in U \mid \operatorname{col}(v) \in Z_{\operatorname{col}(u)}\right\}
$$

We call $A \uplus C$ a feasible solution if it satisfies (i)-(iii), not necessarily maximizing (7). In UFLP-MCC, a facility $u$ of color $i$ gets profit only from clients in $Z(u)$, that is, from clients with a color in $Z_{i}$. Moreover, there can be only one facility of color $i$ and the clients with a color in $Z_{i}$ are only served by facilities of color $i$. Thus, the contribution of a facility $u$ to the goal function of UFLP-MCC is independent from the contributions of other facilities, which makes UFLP-MCC significantly easier than UFLP-MC. We prove the following lemma.

Lemma 5.7. If UFLP-MCC is solvable in $t(k+\ell)$ time, then UFLP-MC is solvable in $2^{O(r \log r)}(t(2 r)+n) \log n$ time, where $r$ is the minimum rank of the client matroids.

To prove Lemma 5.7, we will prove that

(a) any feasible solution to UFLP-MCC is a feasible solution with at least the same profit for UFLP-MC and

(b) for any optimal solution $A \uplus C$ to UFLP-MC, we can sufficiently quickly find a coloring and a partition $Z_{1} \uplus \cdots \uplus Z_{\ell}$ such that $A \uplus C$ is a feasible solution with at least the same profit for UFLP-MCC.

Towards (b), note that we cannot simply try out all partitions and colorings; for example, there are $O\left(r^{n}\right)$ colorings—too many for Lemma 5.7. However, since there is a client matroid of rank $r$, there is an optimal solution to UFLP-MC such that $|C|=k$ and $|A|=\ell$ for some $\ell \leq k \leq r$. Thus, without loss of generality assuming $U=\{1, \ldots, n\}$, the colorings in a $(n, k+\ell)$-perfect hash family as defined below will contain a coloring such that the elements of $A \uplus C$ get pairwise distinct colors: 


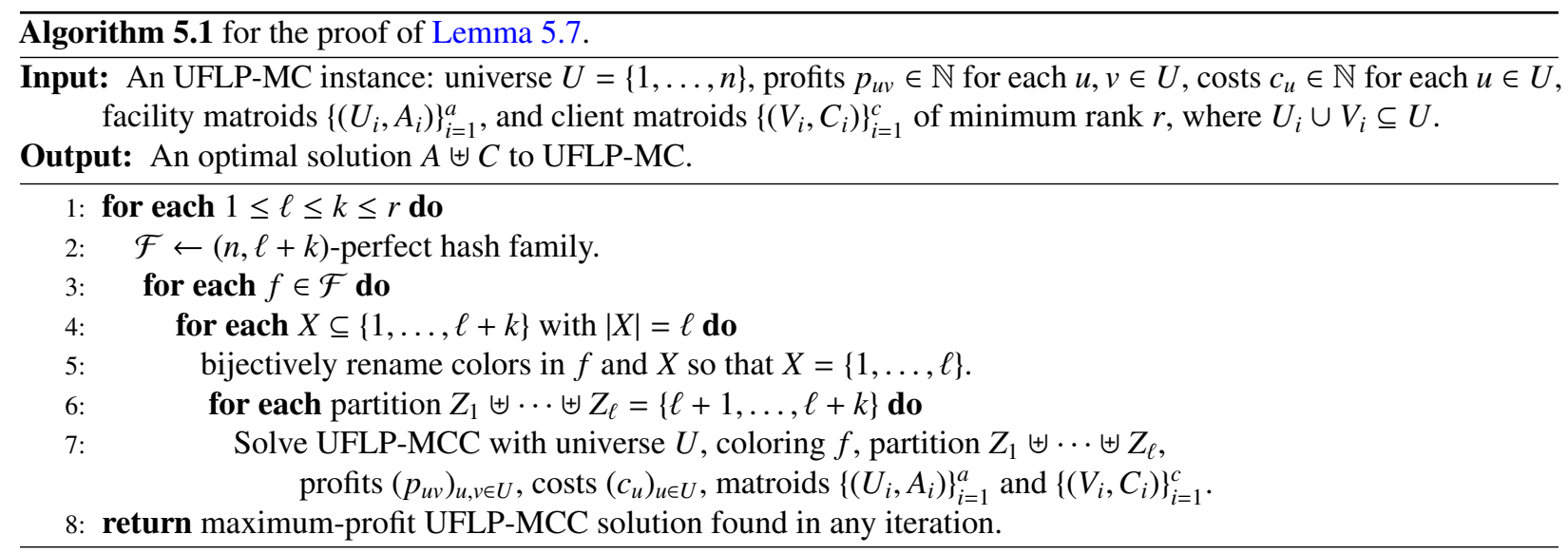

Definition $5.8((n, s)$-perfect hash family [11, Definition 5.17]). An (n,s)-perfect hash family is a set $\mathcal{F}$ of functions $f:\{1, \ldots, n\} \rightarrow\{1, \ldots, s\}$ such that, for any $S \subseteq\{1, \ldots, n\}$ with $|S| \leq s$, there is a function $f \in \mathcal{F}$ injective on $S$.

Proposition 5.9 ([11, Theorem 5.18]). An $(n, s)$-perfect hash family of size $e^{s} s^{O(\log s)} \log n$ can be computed in $e^{s} s^{O(\log s)} n \log n$ time.

In the following, we will prove that Algorithm 5.1 correctly solves UFLP-MC.

Lemma 5.10. Any UFLP-MCC solution A $\uplus C$ is also an UFLP-MC solution with at least the same profit.

Proof. Let $A \uplus C$ be any UFLP-MCC solution. Obviously, it is also feasible for UFLP-MC. Its profit as an UFLP-MCC solution is given by (7) and, denoting $\bar{p}_{u v}:=p_{u v}$ if $v \in Z(u)$ and $\bar{p}_{u v}:=0$ otherwise, is

$$
\sum_{u \in A}\left(-c_{u}+\sum_{v \in C \cap Z(u)} p_{u v}\right)=\sum_{u \in A}\left(-c_{u}+\sum_{v \in C} \bar{p}_{u v}\right)=-\sum_{u \in A} c_{u}+\sum_{v \in C} \sum_{u \in A} \bar{p}_{u v} .
$$

Since, for each $v \in C$, there is exactly one $u \in A$ with $v \in Z(u)$, this is

$$
\leq-\sum_{u \in A} c_{u}+\sum_{v \in C} \max _{u \in A} p_{u v}
$$

which is exactly the cost of $A \uplus C$ as a solution to UFLP-MC as given by (4).

Lemma 5.11. Algorithm 5.1 is correct.

Proof. Let $A \uplus C$ be an optimal solution to UFLP-MC such that $A$ is of minimum size. We show that Algorithm 5.1 outputs a solution to UFLP-MC of equal profit.

Since one of the client matroids has rank $r$, one has $|A|=\ell$ and $|C|=k$ such that $1 \leq \ell \leq k \leq r$. Algorithm 5.1 tries these $\ell$ and $k$ in line 1 . Thus, the $(n, k+\ell)$-perfect hash family $\mathcal{F}$ generated in line 2 contains a function col: $U \rightarrow$ $\{1, \ldots, k+\ell\}$ that is bijective restricted to $A \uplus C$. Algorithm 5.1 tries this function col in line 3. Since $|A|=\ell$ contains elements of pairwise distinct colors, Algorithm 5.1 in line 4 iterates over the color set $X$ of $A$ and renames all colors so that $X=\{1, \ldots, \ell\}$. We get that $A$ contains exactly one element of each color of $X=\{1, \ldots, \ell\}$ and that $C$ contains exactly one element of each color of $\{\ell+1, \ldots, \ell+k\}$.

Now, recall that $U=\{1, \ldots, n\}$ and, for each $v \in C$, let $m(v) \in A$ be the facility with minimum index that serves $v$ with maximum profit, that is,

$$
m(v):=\min \left\{u \in A \mid p_{u v}=\max _{w \in A} p_{w v}\right\} .
$$

Then, for each $u \in A$, there is a $v \in C$ such that $m(v)=u$ : otherwise, we can rewrite the goal function (4) of UFLP-MC as

$$
\sum_{v \in C} \max _{u \in A} p_{u v}-\sum_{u \in A} c_{u}=\sum_{v \in C} p_{m(v), v}-\sum_{u \in A} c_{u},
$$


and removing $u$ from $A$ would yield a solution to UFLP-MC with at least the same profit but smaller $A$, contradicting the minimality of $A$. Thus, for each $i \in\{1, \ldots, \ell\}$, the set $Z_{i}$ of colors of the clients $v$ served by a facility $m(v)$ of color $i$, that is,

$$
Z_{i}:=\{\operatorname{col}(v) \mid v \in C, m(v)=u, \operatorname{col}(u)=i\}
$$

is nonempty. Hence, $Z_{1} \uplus \cdots \uplus Z_{\ell}=\{\ell+1, \ldots, \ell+k\}$ is a partition:

- Equality follows since $C$ contains exactly one element of each color of $\{\ell+1, \ldots, \ell+k\}$ and $m(v)$ is defined for each $v \in C$.

- Pairwise disjointness follows since $m(v)$ for each client $v \in C$ is unique.

Since $Z_{1} \uplus \cdots \uplus Z_{\ell}$ is a partition, Algorithm 5.1 in line 6 iterates over this partition and $A \uplus C$ is a feasible solution to the UFLP-MCC instance in this iteration. We show that its profit as an UFLP-MCC solution, given by (7), is the same as the profit as an UFLP-MC solution, given by (4). To this end, denote

$$
\bar{p}_{u v}:= \begin{cases}p_{u v} & \text { if } m(v)=u, \text { and } \\ 0 & \text { otherwise }\end{cases}
$$

and observe that, for $u \in A$ and $v \in C$, one has $m(v)=u$ if and only if $\operatorname{col}(v) \in Z_{\operatorname{col}(u)}$. By choice in (7), this is if and only if $v \in C \cap Z(u)$. Thus, the cost of $A \uplus C$ as a solution to UFLP-MC is

$$
\sum_{v \in C} \max _{u \in A} p_{u v}-\sum_{u \in A} c_{u}=\sum_{v \in C} \sum_{u \in A} \bar{p}_{u v}-\sum_{u \in A} c_{u}=\sum_{u \in A} \sum_{v \in C \cap Z(u)} p_{u v}-\sum_{u \in A} c_{u},
$$

which is exactly the profit (7) of $A \uplus C$ as a solution to the UFLP-MCC instance given solved in this iteration. Thus, in line 8, Algorithm 5.1 will return an UFLP-MCC solution with at least this profit. By Lemma 5.10, this will be an UFLP-MC solution of at least the same profit. Since $A \uplus C$ is an optimal UFLP-MC solution, we conclude that the solution returned by Algorithm 5.1 is also optimal.

We can now complete the reduction of UFLP-MC to UFLP-MCC.

Proof (of Lemma 5.7). We have shown in Lemma 5.11 that Algorithm 5.1 correctly solves UFLP-MC. It remains to analyze the running time.

The loop in line 1 makes $r \cdot(r+1) / 2$ iterations. Observe that $\ell+k \leq 2 r$. By Proposition 5.9, the perfect hash family in line 2 is computable in $e^{\ell+k} \cdot(\ell+k)^{O(\log (\ell+k))} \cdot n \log n$ time and the loop in line 3 makes $e^{\ell+k} \cdot(\ell+k)^{O(\log (\ell+k))} \cdot \log n$ iterations. Line 5 works in $O(n)$ time, whereas line 7 works in $t(k+\ell)$ time by assumption. There are at most $\ell^{\ell+k}$ variants to chose $X \uplus Z_{1} \uplus \cdots \uplus Z_{\ell}$. Thus, the overall running time of the algorithm is $2^{O(r \log r)}(t(2 r)+n) \log n$.

\subsubsection{One arbitrary facility matroid and one uniform client matroid}

We now prove Theorem 5.3(ii): an algorithm that solves UFLP-MC in $2^{O(r \log r)} \cdot n^{2}$ time if there is only one, yet arbitrary (not necessarily linear) facility matroid and one uniform client matroid of rank $r$. To this end, we show:

Proposition 5.12. UFLP-MCC is solvable in $O\left(\ell n^{2}\right)$ time if there is one facility matroid given as an independence oracle and one client matroid that is uniform.

Then, Theorem 5.3(ii) follows from Proposition 5.12 and Lemma 5.7. To present the algorithm for the proof of Proposition 5.12, we introduce the following notation:

Definition 5.13. For a universe $U$ with coloring col: $U \rightarrow\{1, \ldots, k+\ell\}$, we denote by $U(i):=\{u \in U \mid \operatorname{col}(u)=i\}$ the elements of color $i$. 


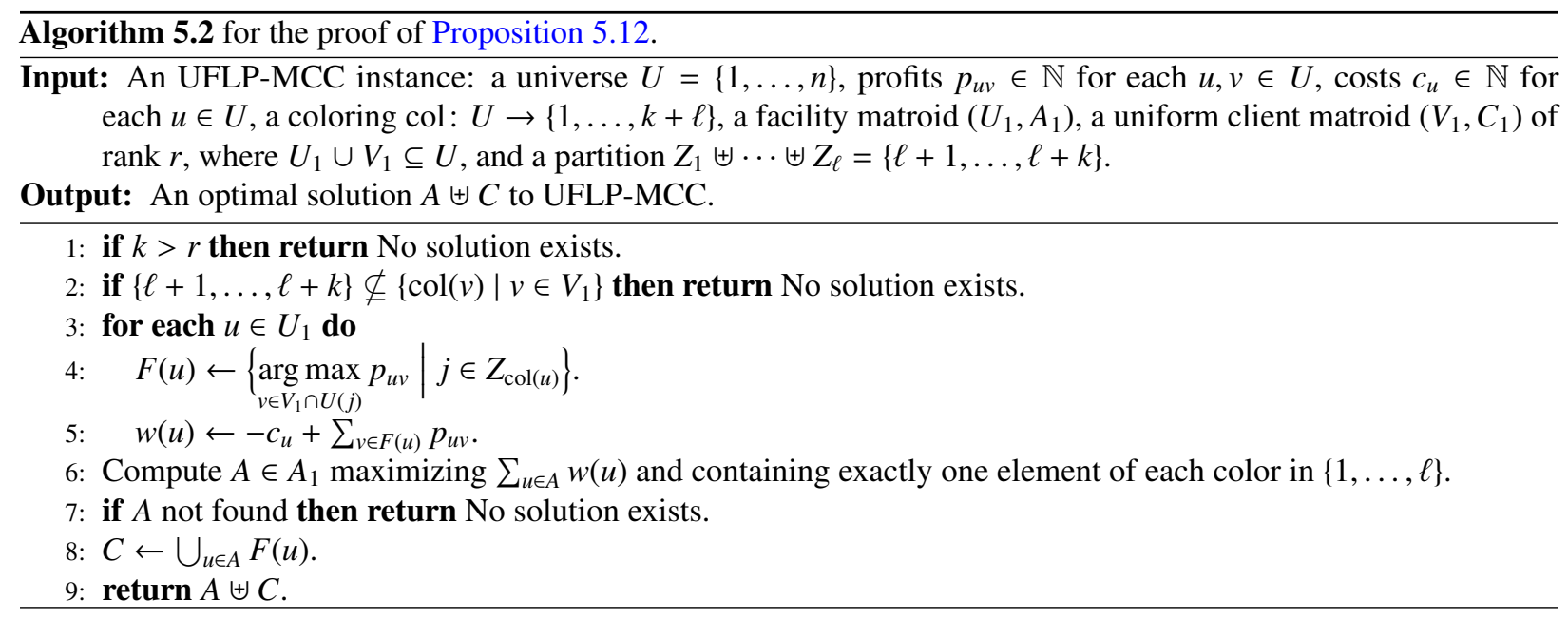

To prove Proposition 5.12, we use Algorithm 5.2, which solves UFLP-MCC as follows. In line 4, for each facility $u$, it computes a set $F(u)$ containing of each color in $Z_{\mathrm{col}(u)}$ exactly one client $v \in V_{1}$ that maximizes $p_{u v}$. The intuition is that if facility $u$ will be part of a solution, then the clients $F(u)$ will follow $u$ into the solution. In line 5, it assigns to each facility $u$ a weight $w(u)$, which is the profit gained from serving the clients in $F(u)$ by $u$ minus the cost for opening facility $u$. Finally, in line 6 , it computes a maximum-weight set $A \in A_{1}$ containing exactly one facility of each color $\{1, \ldots, \ell\}$ and chooses $C:=\bigcup_{u \in A} F(u)$. The crucial point herein is that the set $A$ can be computed as the maximum-weight common independent set of size $\ell$ of two matroids, which can be done in polynomial time [28, Sections 41.3 and 41.3a]. In the following, we prove the correctness and the running time of Algorithm 5.2.

Lemma 5.14. Algorithm 5.2 is correct.

Proof. First, assume that Algorithm 5.2 returns some $A \uplus C$ in line 9. We show that $A \uplus C$ is a feasible solution to the input UFLP-MCC instance. By construction of $A$ in line $6, A \in A_{1}$ and contains exactly one element of each color $\{1, \ldots, \ell\}$. Thus, Problem 5.6(i) is satisfied. Since $Z_{1} \uplus \cdots \uplus Z_{\ell}$ is a partition of $\{\ell+1, \ldots, \ell+k\}$ and line 2 has been passed, $C=\bigcup_{u \in A} F(u)$ for the sets $F(u) \subseteq V_{1}$ computed in line 4 contains exactly one element of each color $\{\ell+1, \ldots, \ell+k\}$ and (ii) is satisfied. Thus, $C \subseteq V_{1}$ and $|C|=k$. Moreover, $k \leq r$ since line 1 has been passed. Thus, since $\left(V_{1}, C_{1}\right)$ is a uniform matroid of rank $r$, it follows that $C \in C_{1}$ and (iii) is satisfied. We conclude that $A \uplus C$ is a feasible solution.

Now assume that there is an optimal solution $A^{*} \uplus C^{*}$ to UFLP-MCC. We show that Algorithm 5.2 returns a solution with the same profit. First, since $C^{*}$ contains exactly one vertex of each color in $\{\ell+1, \ldots, \ell+k\}$ by Problem 5.6(ii), we get $\left|C^{*}\right|=k$. Second, since $C^{*} \in C_{1}$ by (iii), we get $C^{*} \subseteq V_{1}$ and $k=\left|C^{*}\right| \leq r$. Thus, the tests in lines 1 and 2 pass. Thus, line 6 of Algorithm 5.2 computes a set $A \in A_{1}$ containing exactly one element of each color $\{1, \ldots, \ell\}$ (by (i), $A^{*}$ witnesses the existence of such a set), a corresponding set $C=\bigcup_{u \in A} F(u)$ in line 8, and finally returns $A \uplus C$ in line 9, which we already proved to be a feasible solution for UFLP-MCC. It remains to compare the profit of $A \uplus C$ to that of $A^{*} \uplus C^{*}$. To this end, the goal function (7) for $A^{*} \uplus C^{*}$ can be rewritten as

$$
\sum_{u \in A^{*}} w^{\prime}(u) \quad \text { for } \quad w^{\prime}(u):=-c_{u}+\sum_{v \in C^{*} \cap Z(u)} p_{u v}
$$

In comparison, consider the weight $w(u)$ assigned to each $u \in A^{*}$ as in line 5 of Algorithm 5.2. Since, for each $u \in A$ with $\operatorname{col}(u)=i$,

$$
w(u)=-c_{u}+\sum_{v \in F(u)} p_{u v}=-c_{u}+\sum_{j \in Z_{i}} \max _{v \in V_{1} \cap U(j)} p_{u v},
$$

one has $w(u) \geq w^{\prime}(u)$. Since $A$ computed in line 6 maximizes $\sum_{u \in A} w(u)$,

$$
\sum_{u \in A^{*}} w^{\prime}(u) \leq \sum_{u \in A} w(u)=\sum_{u \in A}\left(-c_{u}+\sum_{v \in F(u)} p_{u v}\right)=\sum_{u \in A}\left(-c_{u}+\sum_{v \in C \cap Z(u)} p_{u v}\right),
$$


which is exactly the profit of solution $A \uplus C$ to UFLP-MCC.

Having shown the correctness of Algorithm 5.2, we now analyze its running time.

Lemma 5.15. Algorithm 5.2 can be run in $O\left(\ell n^{2}\right)$ time if the matroid $\left(U_{1}, A_{1}\right)$ is given as an independence oracle.

Proof. Lines 1 to 5, 8, and 9 are easy to implement in $O\left(n^{2}\right)$ time. We show how to execute line 6 efficiently. To this end, consider the partition matroid $\left(U_{1}^{\prime}, B\right)$ in which a subset of $U_{1}$ is independent if it contains at most one element of each color $\{1, \ldots, \ell\}$ and no elements of other colors. Line 6 is then computing a set $A$ of maximum weight and cardinality $\ell$ that is independent in both matroids $\left(U_{1}, A_{1}\right)$ and $\left(U_{1}, B\right)$. This can be done in $O\left(\ell n^{2}\right)$ time [28, Sections 41.3 and $41.3 \mathrm{a}]$.

Lemmas 5.7, 5.14 and 5.15 together finish the proof of Theorem 5.3(ii).

\subsubsection{Facility and client matroids representable over the same field}

In this section, we prove Theorem 5.3(iii): UFLP-MC is fixed-parameter tractable parameterized by the number of matroids and the minimum rank over all client matroids if all matroids are representable over the same field. To this end, we prove the following, which, together with Lemma 5.7, yields Theorem 5.3(iii).

Proposition 5.16. An optimal solution to an UFLP-MCC instance $\mathcal{I}$ can be found in $f(a+c+k+\ell) \cdot \operatorname{poly}(|\mathcal{I}|)$ time if representations of the a facility matroids and c client matroids over the same field $\mathbb{F}_{p^{d}}$ are given for some prime $p$ polynomially upper-bounded by $|\mathcal{I}|$.

The algorithm for Proposition 5.16 is more involved than Algorithm 5.2, which breaks in the presence of client matroids, even a single one: we cannot guarantee that the sets $F(u)$ chosen in line 4 of Algorithm 5.2 are independent in the client matroids or that their union will be. Ideally, one would we able to choose from all possible subsets $F(u) \subseteq Z(u)$ of clients that could be served by $u$, yet there are too many. Here the max intersection representative families that we construct in Section 3.3 come into play: using Theorem 3.11, we compute a family $\widehat{\mathcal{F}}(u)$ so that, if there is any set of clients that can be served by $u$ and that is independent in all client matroids together with the clients served by other facilities, then $\widehat{\mathcal{F}}(u)$ contains at least one such subset yielding at least the same profit. Using Theorem 4.2, we can then compute disjoint unions of these sets maximizing profit. To describe the algorithm, we introduce some notation.

Definition 5.17. For a universe $U$ with coloring col: $U \rightarrow\{1, \ldots, k+\ell\}$ and $U(i)$ as in Definition 5.13, we denote by

$$
\begin{aligned}
U_{A} & :=\bigcup_{i=1}^{\ell} U(i) \\
U_{C} & :=\bigcup_{i=\ell+1}^{\ell+k} U(i)
\end{aligned}
$$$$
\text { is the set of facilities, and }
$$

is the set of clients.

Algorithm 5.3 now solves UFLP-MCC as follows. In line 1, it constructs a multicolored matroid $M_{P}$ that will ensure that any independent set of $k$ facilities and $\ell$ clients fulfills Problem 5.6(i) and (ii). In line 2, it computes a family $\mathcal{M}$ of matroids that contains $M_{P}$ and all facility and client matroids, which are extended so that a set $A \uplus C \subseteq U$ is independent in all of them if and only if $A$ is independent in all facility matroids and $C$ is independent in all client matroids. Now, if one of the matroids in $\mathcal{M}$ has rank less than $k+\ell$, then there is no common independent set of $\ell$ facilities and $k$ clients, which is checked in line 3. The truncation in line 4 thus results in each matroid in $\mathcal{M}$ having rank exactly $k+\ell$, which is needed to apply Theorem 3.11 in line 6 . In line 6 , we construct for each $u \in U_{A}$ with $\operatorname{col}(u)=i$ a max intersection $\left(k+\ell-\left|Z_{i}\right|\right)$-representative $\widehat{\mathcal{F}}(u)$ for the family $\mathcal{F}(u)$ of all sets of clients that could potentially be served by $u$ in a solution. Afterwards, in line 8 , we construct a family of sets, each consisting of one facility $u \in U_{A}$ and a potential client set from $\widehat{\mathcal{F}}(u)$. Finally, in line 9 , we will use Theorem 4.2 to combine $\ell$ of such sets into a set that is independent in all matroids in $\mathcal{M}$ and yields maximum profit. To prove Proposition 5.16, we now show that Algorithm 5.3 is correct and analyze its running time.

Lemma 5.18. Any solution output by Algorithm 5.3 is feasible for UFLP-MCC. 


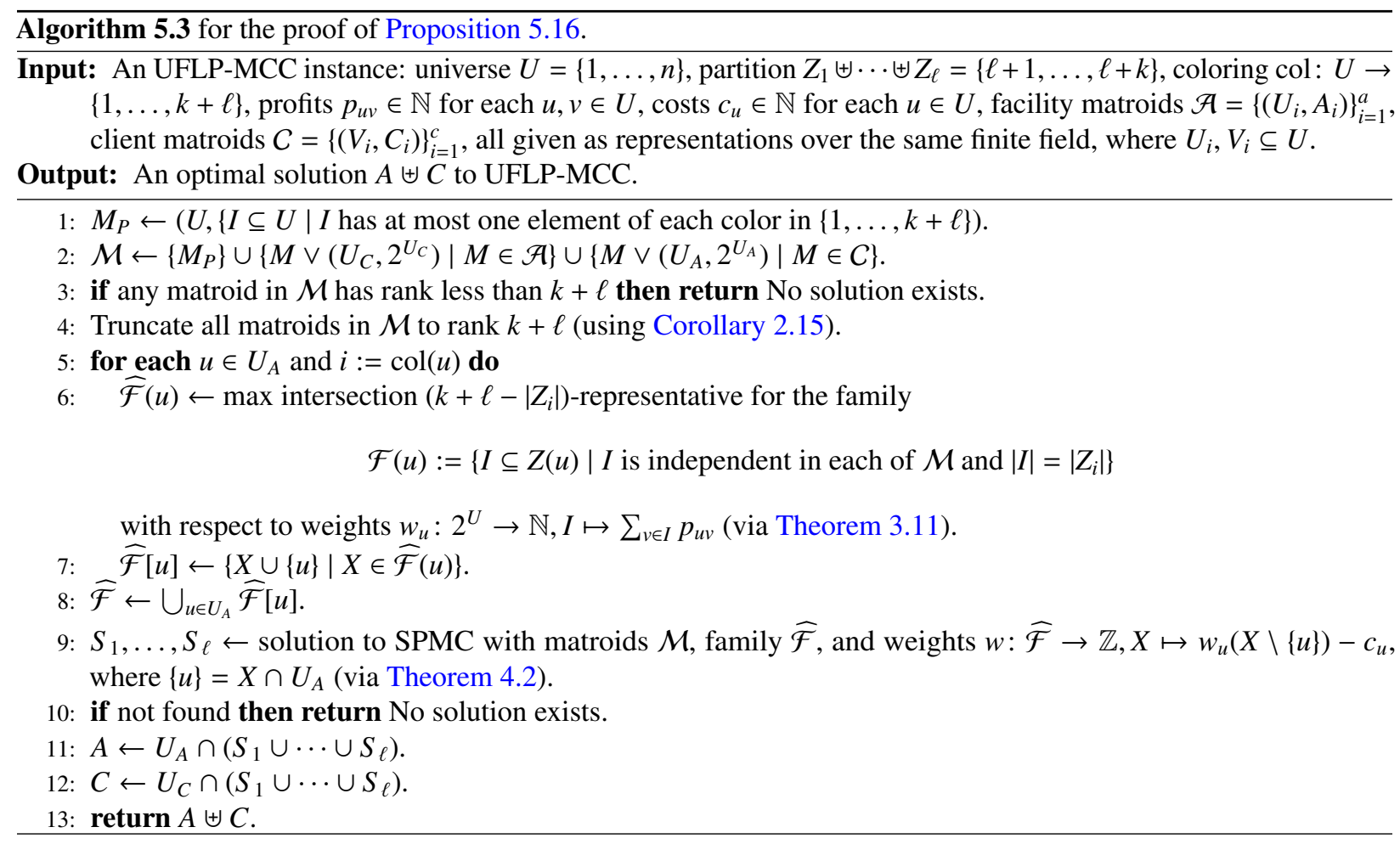

Proof. If Algorithm 5.3 outputs a solution in line 13, then, in line 9 , it founds sets $S_{1}, \ldots, S_{\ell} \in \widehat{\mathcal{F}}$ such that $S=S_{1} \uplus \cdots \uplus S_{\ell}$ is independent in all matroids $\mathcal{M}$. We show that $A \uplus C$ for $A=S \cap U_{A}$ and $C=S \cap U_{C}$ is a feasible solution for UFLP-MCC, that is, it satisfies properties Problem 5.6(i)-(iii).

(i) and (ii): Observe that each set in $\widehat{\mathcal{F}}$ (constructed in line 8) contains exactly one facility $u \in U_{A}$ and $\left|Z_{i}\right|$ elements from $U_{C}$ for $i=\operatorname{col}(u)$. Thus,

$$
|S|=\sum_{i=1}^{\ell}\left|S_{i}\right|=\ell+\sum_{i=1}^{\ell}\left|Z_{i}\right|=\ell+k
$$

since $Z_{1} \uplus \cdots \uplus Z_{\ell}=\{\ell+1, \ldots, \ell+k\}$. Since $S$ is independent in the multicolored matroid $M_{P}$, it follows that $S$ contains exactly one facility and exactly one client of each color. Since $A=S \cap U_{A}$ and $C=S \cap U_{C}$, (i) and (ii) hold.

(iii): Since $A$ is independent in all matroids of $\mathcal{M}$, it is independent in all matroids of $\left\{M \vee\left(U_{C}, 2^{U_{C}}\right) \mid M \in \mathcal{A}\right\}$. Since $A \subseteq U_{A}$ and thus $A \cap U_{C}=\emptyset$, it follows that $A$ is independent in all matroids in $\mathcal{A}$. Analogously, it follows that $C$ is independent in all matroids in $C$.

Lemma 5.19. Given a feasible UFLP-MCC instance, Algorithm 5.3 outputs a solution of maximum profit.

Proof. Let $S=A \uplus C$ be an optimal solution to UFLP-MCC. We show that Algorithm 5.3 outputs a solution of UFLP-MCC with the same profit.

Since $S$ contains exactly one facility of each color in $\{1, \ldots, \ell\}$ by Problem 5.6(i) and exactly one client of each color in $\{\ell+1, \ldots, \ell+k\}$ by (ii), it is independent in the colorful matroid $M_{P}$ constructed in line 1 . Moreover, by (iii), $C$ is independent in all matroids in $C$ and trivially in $\left(U_{C}, 2^{U_{C}}\right)$. Similarly, $A$ is independent in all matroids in $\mathcal{A}$ and $\left(U_{A}, 2^{U_{A}}\right)$. Thus, by Proposition 2.8 about matroid unions, $A \uplus C$ is independent in all matroids in the set $\mathcal{M}$ constructed in line 2. Since $|A \uplus C|=k+\ell$, it follows that each matroid in $\mathcal{M}$ has rank at least $k+\ell$ and line 3 is passed. It follows that after line 4 , all matroids in $\mathcal{M}$ have rank exactly $k+\ell$.

Now, consider an arbitrary facility $u \in A$ and $i:=\operatorname{col}(u)$. For the set $Z(u)$ in $(7)$ and the set $\mathcal{F}(u)$ constructed in line 6, one has $C_{u}:=C \cap Z(u) \in \mathcal{F}(u)$ and

$$
w_{u}\left(C_{u}\right)=\sum_{v \in C_{u}} p_{u v}
$$


Moreover, one has $|A \uplus C|=k+\ell,\left|C_{u}\right|=\left|Z_{i}\right|$, and

$$
A \uplus C=A \uplus \biguplus_{w \in A} C_{w}=\left(A \uplus \biguplus_{w \in A \backslash\{u\}} C_{w}\right) \uplus C_{u} .
$$

Since $\widehat{\mathcal{F}}(u)$ is max intersection $\left(k+\ell-\left|Z_{i}\right|\right)$-representative with respect to $w_{u}$, by Definition 3.1 , there is $C_{u}^{\prime} \in \widehat{\mathcal{F}}(u)$ with $w_{u}\left(C_{u}^{\prime}\right) \geq w_{u}\left(C_{u}\right)$ and such that

$$
A \uplus C^{\prime}=\left(A \uplus \biguplus_{w \in A \backslash\{u\}} C_{w}\right) \uplus C_{u}^{\prime}
$$

is independent in all matroids of $\mathcal{M}$. Consequently, $\{u\} \uplus C_{u}^{\prime} \in \widehat{\mathcal{F}}$ in line 8 and

$$
A \uplus C^{\prime \prime}=\biguplus_{u \in A}\left(\{u\} \uplus C_{u}^{\prime}\right)
$$

is a feasible solution to the SPMC instance in line 9. Thus, Algorithm 5.3 in line 9 finds an optimal SPMC solution $S_{1}, \ldots, S_{\ell} \in \widehat{\mathcal{F}}$ for the weights $w: \widehat{\mathcal{F}} \rightarrow \mathbb{Z}, X \mapsto w_{u}(X \backslash\{u\})-c_{u}$, where $\{u\}=X \cap U_{A}$. It returns $A^{*} \uplus C^{*}$ for $A^{*}=U_{A} \cap\left(S_{1} \uplus \cdots \uplus S_{\ell}\right)$ and $C^{*}=U_{C} \cap\left(S_{1} \uplus \cdots \uplus S_{\ell}\right)$ in line 13, which is a feasible solution for UFLP-MCC by Lemma 5.18. Finally, since each such set $S_{i} \in \widehat{\mathcal{F}}$ consists of one facility $u \in U_{A}$ and $\left|Z_{\mathrm{col}(u)}\right|$ elements of $Z(u)$ with pairwise distinct colors, the profit of $A^{*} \uplus C^{*}$ as a solution to UFLP-MCC given by (7) is

$$
\begin{aligned}
& \sum_{u \in A^{*}}\left(-c_{u}+\sum_{v \in C^{*} \cap Z(u)} p_{u v}\right)=\sum_{j=1}^{\ell} w\left(S_{j}\right) \geq \sum_{u \in A} w\left(\{u\} \uplus C_{u}^{\prime}\right) \\
& =\sum_{u \in A}\left(-c_{u}+w_{u}\left(C_{u}^{\prime}\right)\right) \geq \sum_{u \in A}\left(-c_{u}+w_{u}\left(C_{u}\right)\right)=\sum_{u \in A}\left(-c_{u}+\sum_{v \in C_{u}} p_{u v}\right),
\end{aligned}
$$

which is exactly the profit of the optimal solution $A \uplus C$.

Lemma 5.20. Given representations of all matroids over the same field $\mathbb{F}$, where $\mathbb{F}=\mathbb{F}_{p^{d}}$ such that $p$ is a prime polynomially upper-bounded in the input size, Algorithm 5.3 can be executed in $2^{O(\ell k(a+c))} \cdot \operatorname{poly}(x)$ time, where a is the number of facility matroids, $c$ is the number of client matroids, $k+\ell$ is the number of colors, and $x$ is the input size.

Proof. First, we compute a $((k+\ell) \times n)$-representation $B=\left(b_{i j}\right)$ of the multicolored matroid $M_{P}$ in line 1 of Algorithm 5.3 over $\mathbb{F}$ in $O(n k)$ time: $b_{i j}=1$ if element $j \in U$ has color $i$, and $b_{i j}=0$ otherwise. By Lemma 2.11, we can compute the set $\mathcal{M}$ of matroids and their representations over $\mathbb{F}$ in line 2 in time of a polynomial number of field operations over $\mathbb{F}$.

Due to line 3, all matroids in $\mathcal{M}$ have rank at least $k+\ell$. In line 4 , we use Corollary 2.15 to compute $(k+\ell)$ truncations of all matroids in $\mathcal{M}$ over a field extension $\mathbb{F}^{\prime} \supseteq \mathbb{F}$, in a polynomial number of field operations over $\mathbb{F}$. Herein, $\mathbb{F}^{\prime}=\mathbb{F}_{p^{d^{\prime}}}$ with $d^{\prime}=(k+\ell) \cdot r d \in \operatorname{poly}(n+d)$ since $\ell \leq k \leq n$ and $r$ is the maximum rank of the input matroids.

Let $m=|\mathcal{M}|=a+c+1$. Using Theorem 3.11 with $\gamma=1, \mathcal{H}:=\{\{v\} \mid v \in \mathcal{F}(u)\}$, and weight function $w_{u}: 2^{U} \rightarrow \mathbb{N}$ to implement line 6, we can execute the for-loop starting at line 5 in time of $2^{O((k+\ell) \cdot m)} \cdot n^{2}$ operations over $\mathbb{F}^{\prime}:$ since $\mathcal{H}$ is a 1family and, thus, the partition of any subset of $U$ into sets of $\mathcal{H}$ is unique, the function $w_{u}$ is a inductive union maximizing function generated by $w_{u}(\emptyset)=0$ and the constant-time computable function $\mathbf{g}: \mathbb{N} \times \mathcal{H} \rightarrow \mathbb{N},(k,\{v\}) \mapsto k+p_{u v}(\mathrm{cf}$. Example 3.3).

In line 7, for each $u \in U_{A}$, it holds that $|\widehat{\mathcal{F}}[u]| \leq|\widehat{\mathcal{F}}(u)| \leq\left(\begin{array}{c}(k+\ell) m \\ k m\end{array}\right) \leq 2^{(k+\ell) m}$ by Theorem 3.11. Thus, in line 8, we have $|\widehat{\mathcal{F}}| \leq n 2^{(k+\ell) m}$. Moreover, each set in $\widehat{\mathcal{F}}$ has size at most $k+1$. Therefore, by Theorem 4.2 , line 9 can be executed in time of $2^{O(\ell(k+1) m)} \cdot n \cdot \operatorname{poly}\left(p, d^{\prime}\right)$ operations over $\mathbb{F}^{\prime}$.

Since we initially get the representations of the input matroids over the field $\mathbb{F}_{p^{d}}$ with $p^{d}$ elements, we need at least $d \log p$ bits to encode an element of the field. Thus, $d \log p$ is less than the input size. Therefore, $d$ and $d^{\prime}$ are polynomially bounded by the input size. Since each element of the field $\mathbb{F}_{p^{d^{\prime}}}$ can be encoded using $d^{\prime} \log p$ bits, each field operation over $\mathbb{F}^{\prime}$ (and therefore over $\mathbb{F}$ ) can be executed in poly $\left(d^{\prime} \log p\right.$ ) time, which is polynomial in the input size. Thus, Algorithm 5.3 can be executed in $2^{O(\ell k(a+c))} \cdot \operatorname{poly}(x)$ time.

Proposition 5.16 now follows from Lemmas 5.18 to 5.20. Finally, Theorem 5.3(iii) follows from Proposition 5.16 and Lemma 5.7. 


\section{Conclusion}

The complexity of UFLP-MC seems to be determined by the client matroids: it is fixed-parameter tractable parameterized by the minimum rank of the client matroids in case when the facility matroid is arbitrary and the client matroid is uniform, or when all matroids are linear. The problem becomes W[1]-hard for general client matroids, even without facility matroids. It would be interesting to settle the complexity of UFLP-MC with one arbitrary facility matroid parameterized by the rank of a single linear client matroid.

We point out that the algorithms in Theorem 5.3(ii) and (iii) are easy to implement: the construction of perfect hash families using Proposition 5.9 can be replaced by coloring the universe uniformly at random with $k+\ell$ colors [4] and the truncation of matroids using Corollary 2.15, involving large field extensions and generation of irreducible polynomials, can be replaced by a very simple randomized algorithm that does not enlarge fields [25, Proposition 3.7]. Doing so, when aiming for an error probability of at most $\varepsilon \in(0,1)$, the asymptotic running time of our algorithms increases by a factor $\ln (1 / \varepsilon)$.

For future research, we point out that our algorithm for Theorem 5.3(ii) works in polynomial space, whereas Theorem 5.3(iii) requires exponential space due to Theorems 3.11 and 4.2. It is interesting whether this is avoidable. Moreover, given that approximation algorithms are known for UFLP without matroid constraints [2], for the minimization variant of UFLP with a single facility matroid $[19,30]$, as well as for other optimization problems under matroid constraints [9, 13, 22], it is canonical to study approximation algorithms for UFLP-MC.

Acknowledgments. We thank F. V. Fomin, F. Panolan, and the anonymous referees for valuable input. This study was initiated at the 7th annual research retreat of the Algorithmics and Computational Complexity group of TU Berlin, Darlingerode, Germany, March 18th-23rd, 2018.

Funding. René van Bevern was supported by Russian Foundation for Basic Research (RFBR) grant 18-501-12031 NNIO_a. Oxana Yu. Tsidulko was supported by RFBR grant 18-31-00470 mol_a.

\section{References}

[1] Aardal, K., van den Berg, P.L., Gijswijt, D., Li, S.: Approximation algorithms for hard capacitated $k$-facility location problems. European Journal of Operational Research 242(2), 358-368 (2015). doi:10.1016/j.ejor.2014.10.011.

[2] Ageev, A.A., Sviridenko, M.I.: An 0.828-approximation algorithm for the uncapacitated facility location problem. Discrete Applied Mathematics 93(2), 149-156 (1999). doi:10.1016/S0166-218X(99)00103-1.

[3] Aho, A.V., Hopcroft, J.E., Ullman, J.D.: Data Structures and Algorithms. Addison-Wesley (1983). doi:10.1002/bimj.4710260406.

[4] Alon, N., Yuster, R., Zwick, U.: Color-coding. Journal of the ACM 42(4), 844-856 (1995). doi:10.1145/210332.210337.

[5] van Bevern, R., Tsidulko, O.Yu., Zschoche, P.: Fixed-parameter algorithms for maximum-profit facility location under matroid constraints. In: P. Heggernes (ed.) CIAC 2019, pp. 62-74. Lecture Notes in Computer Science, Springer International Publishing (2019). doi:10.1007/978-3-030-17402-6_6.

[6] Bonnet, É., Paschos, V.T., Sikora, F.: Parameterized exact and approximation algorithms for maximum $k$-set cover and related satisfiability problems. RAIRO-Theoretical Informatics and Applications 50(3), 227-240 (2016). doi:10.1051/ita/2016022.

[7] Briggs, P., Torczon, L.: An efficient representation for sparse sets. ACM Letters on Programming Languages and Systems 2(1-4), 59-69 (1993). doi:10.1145/176454.176484.

[8] Bunch, J.R., Hopcroft, J.E.: Triangular factorization and inversion by fast matrix multiplication. Mathematics of Computation 28(125), 231-231 (1974). doi:10.1090/s0025-5718-1974-0331751-8.

[9] Calinescu, G., Chekuri, C., Pál, M., Vondrák, J.: Maximizing a monotone submodular function subject to a matroid constraint. SIAM Journal on Computing 40(6), 1740-1766 (2011). doi:10.1137/080733991.

[10] Chen, D.Z., Li, J., Liang, H., Wang, H.: Matroid and knapsack center problems. Algorithmica 75(1), 27-52 (2016). doi:10.1007/s00453-015-0010-1.

[11] Cygan, M., Fomin, F.V., Kowalik, L., Lokshtanov, D., Marx, D., Pilipczuk, M., Pilipczuk, M., Saurabh, S.: Parameterized Algorithms. Springer (2015). doi:10.1007/978-3-319-21275-3.

[12] Fellows, M.R., Fernau, H.: Facility location problems: A parameterized view. Discrete Applied Mathematics 159(11), 1118-1130 (2011). doi:10.1016/j.dam.2011.03.021. 
[13] Filmus, Y., Ward, J.: The power of local search: Maximum coverage over a matroid. In: C. Dürr, T. Wilke (eds.) STACS 2012, Leibniz International Proceedings in Informatics (LIPIcs), vol. 14, pp. 601-612. Schloss Dagstuhl-Leibniz-Zentrum fuer Informatik, Dagstuhl, Germany (2012). doi:10.4230/LIPIcs.STACS.2012.601.

[14] Fomin, F.V., Lokshtanov, D., Panolan, F., Saurabh, S.: Efficient computation of representative families with applications in parameterized and exact algorithms. Journal of the ACM 63(4), 29:1-29:60 (2016). doi:10.1145/2886094.

[15] Fomin, F.V., Lokshtanov, D., Panolan, F., Saurabh, S.: Representative families of product families. ACM Transactions on Algorithms 13(3), 36:1-36:29 (2017). doi:10.1145/3039243.

[16] Goyal, P., Misra, N., Panolan, F., Zehavi, M.: Deterministic algorithms for matching and packing problems based on representative sets. SIAM Journal on Discrete Mathematics 29(4), 1815-1836 (2015). doi:10.1137/140981290.

[17] Kalhan, S.: The Capacitated Matroid Median Problem. Ph.D. thesis, University of Waterloo, Waterloo, Ontario, Canada (2018).

[18] Karp, R.M.: Reducibility among combinatorial problems. In: Complexity of Computer Computations, pp. 85-103. The IBM Research Symposia Series, Plenum Press, New York (1972). doi:10.1007/978-1-4684-2001-2_9.

[19] Krishnaswamy, R., Kumar, A., Nagarajan, V., Sabharwal, Y., Saha, B.: Facility location with matroid or knapsack constraints. Mathematics of Operations Research 40(2), 446-459 (2015). doi:10.1287/moor.2014.0678.

[20] Laporte, G., Nickel, S., Saldanha da Gama, F. (eds.): Location Science. Springer (2015). doi:10.1007/978-3-319-13111-5.

[21] Lawler, E.: Combinatorial Optimization—Networks and Matroids. Holt, Rinehart and Winston, New York (1976).

[22] Lee, J., Sviridenko, M., Vondrák, J.: Matroid matching: The power of local search. SIAM Journal on Computing 42(1), 357-379 (2013). doi:10.1137/11083232X.

[23] Lokshtanov, D., Misra, P., Panolan, F., Saurabh, S.: Deterministic truncation of linear matroids. ACM Transactions on Algorithms 14(2), 14:1-14:20 (2018). doi:10.1145/3170444.

[24] Marx, D.: Parameterized complexity and approximation algorithms. The Computer Journal 51(1), 60-78 (2008). doi:10.1093/comjnl/bxm048.

[25] Marx, D.: A parameterized view on matroid optimization problems. Theoretical Computer Science 410(44), 4471-4479 (2009). doi:10.1016/j.tcs.2009.07.027.

[26] Oxley, J.G.: Matroid Theory. Oxford University Press (1992).

[27] Panolan, F., Saurabh, S.: Matroids in parameterized complexity and exact algorithms. In: M.Y. Kao (ed.) Encyclopedia of Algorithms, pp. 1203-1205. Springer (2016). doi:10.1007/978-1-4939-2864-4.

[28] Schrijver, A.: Combinatorial optimization: polyhedra and efficiency, Algorithms and Combinatorics, vol. 24. Springer (2003).

[29] Shoup, V.: New algorithms for finding irreducible polynomials over finite fields. Mathematics of Computation 54, 435-447 (1990). doi:10.1090/S0025-5718-1990-0993933-0.

[30] Swamy, C.: Improved approximation algorithms for matroid and knapsack median problems and applications. ACM Transactions on Algorithms 12(4), 49:1-49:22 (2016). doi:10.1145/2963170.

[31] Zehavi, M.: Mixing color coding-related techniques. In: N. Bansal, I. Finocchi (eds.) ESA 2015, Lecture Notes in Computer Science, vol. 9294, pp. 1037-1049. Springer (2015). doi:10.1007/978-3-662-48350-3_86. 